\title{
Global Solutions to the Compressible Euler Equations with Geometrical Structure
}

\author{
Gui-Qiang Chen', James Glimm² \\ ${ }^{1}$ Department of Mathematics, Northwestern University, Evanston, Illinois 60208, USA \\ E-mail: gqchen@math nwu edu \\ 2 Department of Applied Mathematics and Statistics, State University of New York, Stony Brook, \\ New York 11794, USA. \\ E-mail: glimm@ams sunysb edu
}

Received: 17 October 1995 / Accepted: 30 January 1996

\begin{abstract}
We prove the existence of global solutions to the Euler equations of compressible isentropic gas dynamics with geometrical structure, including transonic nozzle flow and spherically symmetric flow. Due to the presence of the geometrical source terms, the existence results themselves are new, especially as they pertain to radial flow in an unbounded region, $|\vec{x}| \geqq 1$, and to transonic nozzle flow. Arbitrary data with $L^{\infty}$ bounds are allowed in these results. A shock capturing numerical scheme is introduced to compute such flows and to construct approximate solutions. The convergence and consistency of the approximate solutions generated from this scheme to the global solutions are proved with the aid of a compensated compactness framework.
\end{abstract}

\section{Contents}

1. Introduction . . . . . . . . . . . . . . . . . . . . . . 153

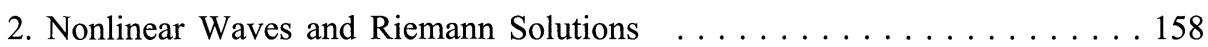

3. Steady-State Solutions . . . . . . . . . . . . . . . . . . . . . 162

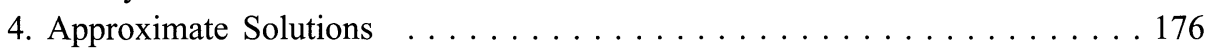

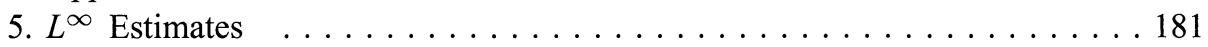

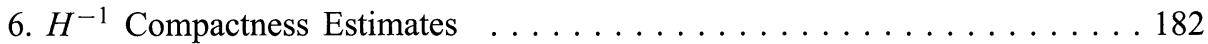

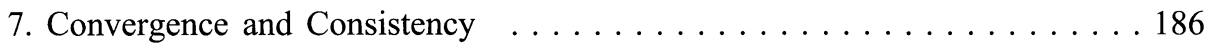

8. Transonic Nozzle Flow: Proof of Theorem A . . . . . . . . . . . . . . 189

9. Spherically Symmetric Flow: Proof of Theorem B . . . . . . . . . . . 189

References

\section{Introduction}

We develop new mathematical existence theory and numerical schemes for global discontinuous solutions to the Euler equations of compressible isentropic gas 
dynamics with large initial data and with geometrical structure. The compressible Euler equations are of the following conservation form:

$$
\left\{\begin{array}{l}
\rho_{t}+\nabla \cdot \vec{m}=0, \\
\vec{m}_{t}+\nabla \cdot\left(\frac{\vec{m} \otimes \vec{m}}{\rho}\right)+\nabla p=0,
\end{array}\right.
$$

where $\rho, \vec{m}$, and $p$ are the density, the momentum, and the pressure of the gas, respectively. On the non-vacuum state, $\vec{u}=\vec{m} / \rho$ is the velocity. For polytropic gas, $p(\rho)=\rho^{\gamma} / \gamma$, where the adiabatic exponent $\gamma$ is restricted to the interval $(1,5 / 3]$, as is usual for gases.

Our results are new for the problem of spherically symmetric flow in an unbounded domain $(|\vec{x}| \geqq 1)$ and for transonic nozzle flow: existence of global discontinuous solutions for general initial data in $L^{\infty}$. The central difficulty in the unbounded domain is the reflection of waves from infinity and their strengthening as they move radially inward. The central difficulty for the transonic nozzle flow lies in the associated steady-state equations, which change type from elliptic to hyperbolic at the sonic point; such steady state solutions are fundamental building blocks in our approach and in early work on nozzle flow.

The Cauchy problem models transonic nozzle flow through a variable-area duct (cf. [EM, GL, GM, L1-L3, CF, Wh]). In [L1] the existence of global solutions of this problem was obtained by first incorporating the steady-state building blocks with the random choice method [G1], provided that the initial data have small total variation and are bounded away from both sonic and vacuum states. A generalized random choice method was introduced to compute transient gas flows in a Laval nozzle in [GL, GM]. A mathematical analysis of the qualitative behavior of nonlinear waves for nozzle flow was given in [L3]. In this paper we introduce a Godunov shock capturing scheme to obtain $L^{\infty}$ estimates and compensated compactness of the corresponding approximate solutions. Our method incorporates natural building blocks from Riemann solutions and steady-state solutions. Such estimates lead to the convergence of the approximate solutions and to an existence theory of global weak entropy solutions for measurable initial data in $L^{\infty}$.

The initial-boundary problem is motivated by many important physical problems such as flow in a jet engine inlet manifold and stellar dynamics including supernovae formation. A global weak entropy solution with spherical symmetry was constructed in $[\mathrm{MU}]$ for the isothermal case $\gamma=1$ and the local existence of such a weak solution for the general case $1<\gamma \leqq 5 / 3$ was also discussed in [MT]. A theorem has also been established for the general case in [C3] to ensure the existence of $L^{\infty}$ spherically symmetric weak solutions in the large for a class of $L^{\infty}$ Cauchy data of arbitrarily large amplitude, which model outgoing blast waves and large-time asymptotic solutions.

In Sects. 2-4 we develop a first order Godunov shock capturing scheme, with piecewise constant building blocks replaced by piecewise steady ones. The main point is to use the steady-state solutions, which incorporate geometrical source terms, to modify the wave strengths in the Riemann solutions. This construction yields better approximate solutions, and permits uniform $L^{\infty}$ bounds. There are two technical difficulties which we overcome to achieve this goal, both due to transonic phenomena. One is that no smooth steady-state solution exists in each cell in general. This problem is easily solved by introducing a standing shock at the center 
of the cell, as discussed in Sect. 2. The other is that the constructed steady-state solution in each cell must satisfy the following requirements:

(a) The oscillation of the steady-state solution around the Godunov value must be of the same order as the cell length to obtain the $L^{\infty}$ estimate for the convergence arguments;

(b) The difference between the average of the steady-state solution over each cell and the Godunov value must be higher than first order in the cell length to ensure the consistency of the corresponding approximate solutions with the Euler equations.

These requirements are satisfied by smooth steady-state solutions bounded away from the sonic state in the cell. The general case must include the transonic steadystate solutions. The sonic difficulty is overcome, as in experimental physics, by introducing an additional standing shock with continuous mass and by adjusting its left state and right state in the density and its location to control the growth of the density. These requirements also enable us to make $H^{-1}$ compactness estimates for corresponding entropy dissipation measures to deduce the strong convergence of the approximate solutions with the aid of the compactness framework (see [C1, C2]).

We rewrite (1.1) with geometrical structure as

$$
\left\{\begin{array}{l}
\rho_{t}+m_{x}=a(x) m, \\
m_{t}+\left(\frac{m^{2}}{\rho}+p(\rho)\right)_{x}=a(x) \frac{m^{2}}{\rho}
\end{array}\right.
$$

or in a compact form:

$$
v_{t}+f(v)_{x}=a(x) g(v),
$$

where $m$ is the momentum of the gas, $a(x)$ is a $C^{2}$ function in the region of $x=|\vec{x}|$ under consideration, $v=(\rho, m)^{\top}, f(v)=\left(m, m^{2} / \rho+p(\rho)\right)^{\top}$, and $g(v)=$ $\left(m, m^{2} / \rho\right)^{\top}$. The function $a(x)$ can be represented by

$$
a(x)=-A^{\prime}(x) / A(x) \quad \text { with } A(x)=e^{-\int^{x} a(y) d y} .
$$

The function $A(x)$ represents the cross-sectional area at $x$ in a variable-area duct for transonic nozzle flow, $A(x)=2 \pi x$ for cylindrically symmetric flow, and $A(x)=$ $4 \pi x^{2}$ for spherically symmetric flow. For cylindrical and spherical flow, we impose reflecting boundary conditions at $x=1$ to exclude the singularity at the origin, but, as a principal new result of this paper, we are able to handle successfully the difficulties at infinity.

We consider the Cauchy problem:

$$
\left.v\right|_{t=0}=v_{0}(x)
$$

and the initial-boundary value problem:

$$
\left\{\begin{array}{l}
\left.v\right|_{t=0}=v_{0}(x) \\
\left.m\right|_{x=1}=0
\end{array}\right.
$$

with the initial data $v_{0}(x) \in L^{\infty}$.

A pair of mappings $(\eta, q): R^{2} \rightarrow R^{2}$ is called an entropy-entropy flux pair [La1] if it satisfies an identity

$$
\nabla q=\nabla \eta \nabla f
$$


Furthermore, if, for any fixed $\frac{m}{\rho} \in(-\infty, \infty), \eta$ vanishes on the vacuum $\rho=0$, then $\eta$ is called a weak entropy. For example, the mechanical energy-energy flux pair

$$
\eta_{*}=\frac{1}{2} \frac{m^{2}}{\rho}+\frac{1}{\gamma(\gamma-1)} \rho^{\gamma}, \quad q_{*}=m\left(\frac{1}{2} \frac{m^{2}}{\rho^{2}}+\frac{\rho^{\gamma-1}}{\gamma-1}\right)
$$

is a strictly convex weak entropy-entropy flux pair. One can prove that, for $0 \leqq$ $\rho \leqq C,\left|\frac{m}{\rho}\right| \leqq C$,

$$
|\nabla \eta| \leqq \text { const. }
$$

and

$$
\left|\nabla^{2} \eta(r, r)\right| \leqq \text { const. } \nabla^{2} \eta_{*}(r, r),
$$

for any weak entropy $\eta$, where $r$ is any vector and the constant is independent of $r$.

Definition 1.1. A pair of measurable functions $v(x, t)=(\rho(x, t), m(x, t))$ is called $a$ global weak entropy solution of the Cauchy problem (1.3) and (1.5) if, for any test function $\phi \in C_{0}^{1}(\Omega)$ with $\Omega \subset \mathbf{R}_{+}^{2} \equiv \mathbf{R} \times \mathbf{R}_{+}$,

$$
\iint_{\Omega}\left(v \phi_{t}+f(v) \phi_{x}+a(x) g(v) \phi\right) d x d t+\int_{\operatorname{supp} \phi(\cdot, 0)} v_{0}(x) \phi(x, 0) d x=0 ;
$$

and, along any shock wave with left state $v_{-}$, right state $v_{+}$, and speed $\sigma$,

$$
\sigma\left(\eta\left(v_{+}\right)-\eta\left(v_{-}\right)\right)-\left(q\left(v_{+}\right)-q\left(v_{-}\right)\right) \geqq 0,
$$

for any convex weak entropy-entropy flux pair $(\eta, q)$ It is called a global weak entropy solution of the initial-boundary problem (1.3) and (1.6) provided that

$$
\frac{1}{\varepsilon} \int_{1}^{1+\varepsilon} m(x, t) d x \stackrel{*}{\rightarrow} 0, \quad \text { in } L_{\mathrm{loc}}^{\infty}\left(\mathbf{R}_{+}\right), \quad \text { as } \varepsilon \rightarrow 0 ;
$$

and, for any convex weak entropy pair $(\eta, q)$ and any $C^{1}$ test function $\phi$ with $\operatorname{supp} \phi \subset(1, \infty) \times \mathbf{R}_{+}$, both (1.11) and (1.12) hold.

For the initial-boundary problem for the compressible Euler equations (1.1) with

$$
\left\{\begin{array}{l}
\left.\vec{m} \cdot \vec{x}\right|_{|\vec{x}|=1}=0, \\
\left.(\rho, \vec{m})\right|_{t=0}=\left(\rho_{0}(\vec{x}), \vec{m}_{0}(\vec{x})\right) \equiv\left(\rho_{0}(|\vec{x}|), m_{0}(|\vec{x}|) \frac{\vec{x}}{|\vec{x}|}\right), \quad|\vec{x}| \geqq 1,
\end{array}\right.
$$

we introduce the following conventional notion of weak entropy solution.

Definition 1.2. A measurable vector function $(\rho(\vec{x}, t), \vec{m}(\vec{x}, t))$ is called a global weak entropy solution of the initial-boundary problem (1.1) and (1.14) provided that

(1) The vector function $(\rho(\vec{x}, t), \vec{m}(\vec{x}, t))$ satisfies the Euler equations (1.1) in the sense of distributions with respect to the test function space $\left\{\phi \in C_{0}^{\infty}(\{|\vec{x}|>\right.$ $\left.\left.1\} \times \mathbf{R}_{+}\right) \mid \phi(\vec{x}, t)=\phi(|\vec{x}|, t)\right\}$.

$$
\frac{1}{\varepsilon} \int_{1}^{1+\varepsilon} \vec{m}(\vec{x}, t) \cdot \frac{\vec{x}}{x} d x \stackrel{*}{\rightarrow} 0, \quad \text { as } \varepsilon \downarrow 0, \quad \text { in } L_{\mathrm{loc}}^{\infty}\left(S^{1} \times \mathbf{R}_{+}\right) ;
$$


(3) Along any shock wave propagating in the direction $\vec{v} \in \mathbf{R}^{N},|\vec{v}|=1$, with left and right states $\left(\rho_{ \pm}, \vec{m}_{ \pm}\right)$and speed $s=s\left(\rho_{-}, \rho_{+}, \vec{m}_{-}, \vec{m}_{+} ; \vec{v}\right)$,

$$
\begin{aligned}
& s\left\{\rho_{+}\left(\frac{\left|\vec{m}_{+}\right|^{2}}{2 \rho_{+}^{2}}+e_{+}\right)-\rho_{-}\left(\frac{\left|\vec{m}_{-}\right|^{2}}{2 \rho_{-}^{2}}+e_{-}\right)\right\} \\
& \quad-\vec{v} \cdot\left\{\vec{m}_{+}\left(\frac{\left|\vec{u}_{+}\right|^{2}}{2 \rho_{+}}+e_{+}+\frac{p_{+}}{\rho_{+}}\right)-\vec{m}_{-}\left(\frac{\left|\vec{u}_{-}\right|^{2}}{2 \rho_{-}}+e_{-}+\frac{p_{-}}{\rho_{-}}\right)\right\} \geqq 0,
\end{aligned}
$$

where $e=\frac{\rho^{\gamma-1}}{\gamma(\gamma-1)}$ is the internal energy.

In these definitions, the entropy conditions (1.12) and (1.16) are equivalent to the corresponding Lax entropy conditions along the shock waves (cf. [Lal, D2, $\mathrm{Sm}]$ ).

Our main results of this paper are included in the following theorems. For the Cauchy problem (1.3)-(1.5), which models the transonic nozzle flow, we have

Theorem A. Assume that $A(x)$ is a $C^{2}$ function bounded away from zero for all $x \in \mathbf{R}$ and the initial data satisfy

$$
0 \leqq \rho_{0}(x) \leqq C_{0}, \quad\left|\frac{m_{0}(x)}{\rho_{0}(x)}\right| \leqq C_{0},
$$

for some $C_{0}>0$. Then there exists a global weak entropy solution $(\rho(x, t), m(x, t))$ of the Cauchy problem (1.3)-(1.5) in the sense of Definition 1.1 satisfying

$$
0 \leqq \rho(x, t) \leqq C(T), \quad\left|\frac{m(x, t)}{\rho(x, t)}\right| \leqq C(T),
$$

for some $C(T) \geqq C_{0}$ in the region $\mathbf{R} \times[0, T]$ for any fixed $T \in(0, \infty)$.

For the initial-boundary problem (1.1) and (1.14), which models the spherically symmetric flow, we have

Theorem B. Assume that the initial data are of the form (1.14) with $\left(\rho_{0}(x), m_{0}(x)\right)$ $\in L^{\infty}(\{x \geqq 1\})$ satisfying (1.17). Then there exists a global weak entropy solution $(\rho(\vec{x}, t), \vec{m}(\vec{x}, t))$ of the initial-boundary problem (1.1) and (1.14) in the sense of Definition 1.2, which takes the form

$$
(\rho, \vec{m})(\vec{x}, t)=\left(\rho(x, t), m(x, t) \frac{\vec{x}}{x}\right),
$$

with $(\rho(x, t), m(x, t)) \in L^{\infty}\left(\{x \geqq 1\} \times \mathbf{R}_{+}\right)$satisfying $(1.18)$.

Note that it is sufficient to show that $v(x, t)=(\rho(x, t), m(x, t))$ is a global weak entropy solution of the initial-boundary problem (1.3) and (1.6), or the Cauchy problem (1.3) and (1.5), in the sense of Definition 1.1. To achieve these results, we also apply a compensated compactness framework (7.1)-(7.2) (Sect. 7) in [Cl, C2] (also see [DC1-2, Di]): uniform boundedness (7.1) of the approximate solutions $\left(\rho^{h}(x, t), m^{h}(x, t)\right)$ and $H^{-1}$ compactness (7.2) of the corresponding entropy dissipation measures imply the strong convergence of the approximate solutions $\left(\rho^{h}(x, t), m^{h}(x, t)\right)$ to the global weak entropy solution $(\rho(x, t), m(x, t)) \in$ 
$L^{\infty}$ of the initial-boundary problem (1.3) and (1.6) and the Cauchy problem (1.3) and (1.5), respectively, almost everywhere with the same property (7.1). The importance of this framework is that it takes the vacuum into account in correct physical variables $(\rho, m)$ near the vacuum, rather than $(\rho, u)$ that is physically incorrect on the vacuum. This framework was proved in [Di] for the case $\gamma=1+\frac{2}{2 m+1}, m \geqq 2$ integers, and in [C1, C2, DC1] for the general case of gases $1<\gamma \leqq 5 / 3$. Further discussions on this framework for other cases can be found in [LP].

In Sect. 2 we construct two solutions which will serve as building blocks for our construction: Riemann solutions for the homogeneous system of gas dynamics and (exact and approximate) steady-state solutions for the inhomogeneous system (1.2). We discuss their basic properties in Sects. 2 and 3.

Section 4 is devoted to the construction of the shock capturing scheme and the corresponding approximate solution of the problems (1.5) and (1.6) for (1.3). Some basic properties of the approximate solutions are discussed. It is proved in Sect. 5 and Sect. 6 that the approximate solutions satisfy the compensated compactness framework $(7.1)-(7.2)$ (see $[\mathrm{C} 1, \mathrm{C} 2])$. The existence theory is established in Sect. 7.

Then the existence theory is applied to the transonic nozzle flow in Sect. 8 (Theorem A) and the spherically symmetric flow in Sect. 9 (Theorem B).

By the methods developed here, we have also proved existence for the initial boundary value problem that models the cylindrically symmetric flow in the unbounded domain $|\vec{x}| \geqq 1$ (see [CG]). The ideas developed here have been also applied to solving the compressible Euler-Poisson equations with geometrical structure that model semiconductor devices and biological channel proteins (see $[\mathrm{CW}])$.

\section{Nonlinear Waves and Riemann Solutions}

In this section we first review some nonlinear waves in gas dynamics and construct Riemann solutions for the homogeneous system of gas dynamics. Then we discuss their basic properties for use in subsequent developments.

2.1. Shock Waves and Rarefaction Waves for 1-D Gas Dynamics. Consider the Riemann problem for the one-dimensional system of isentropic gas dynamics:

$$
\left\{\begin{array}{l}
\rho_{t}+m_{x}=0, \\
m_{t}+\left(\frac{m^{2}}{\rho}+p(\rho)\right)_{x}=0, \quad p(\rho)=\rho^{\gamma} / \gamma,
\end{array}\right.
$$

with

$$
(\rho, m)= \begin{cases}\left(\rho_{-}, m_{-}\right), & x<x_{0}, \\ \left(\rho_{+}, m_{+}\right), & x>x_{0},\end{cases}
$$

where $x_{0} \in(-\infty, \infty), \rho_{ \pm} \geqq 0$ and $m_{ \pm}$are constants satisfying $\left|\frac{m_{ \pm}}{\rho_{ \pm}}\right|<\infty$.

The eigenvalues of the system are

$$
\lambda_{1}=\frac{m}{\rho}-c \equiv c(M-1), \quad \lambda_{2}=\frac{m}{\rho}+c \equiv c(M+1),
$$


where the sound speed $c=\rho^{\theta}$, the Mach number $M=\frac{m}{\rho c}$, and $\theta=\frac{\gamma-1}{2}$. Corresponding Riemann invariants are

$$
w=\frac{m}{\rho}+\frac{\rho^{\theta}}{\theta} \equiv \frac{c}{\theta}(\theta M+1), \quad z=\frac{m}{\rho}-\frac{\rho^{\theta}}{\theta} \equiv \frac{c}{\theta}(\theta M-1) .
$$

Any discontinuity in the weak solutions to (2.1) must satisfy the RankineHugoniot condition:

$$
\sigma\left(v-v_{0}\right)=f(v)-f\left(v_{0}\right),
$$

where $\sigma$ is the propagation speed of the discontinuity, and $v_{0}=\left(\rho_{0}, m_{0}\right)$ and $v=$ $(\rho, m)$ are the corresponding left state and right state. This means that

$$
\left\{\begin{array}{l}
m-m_{0}=\frac{m_{0}}{\rho_{0}}\left(\rho-\rho_{0}\right) \pm \sqrt{\frac{\rho}{\rho_{0}} \frac{p(\rho)-p\left(\rho_{0}\right)}{\rho-\rho_{0}}}\left(\rho-\rho_{0}\right), \\
\sigma=\frac{m-m_{0}}{\rho-\rho_{0}}=\frac{m_{0}}{\rho_{0}} \pm \sqrt{\frac{\rho}{\rho_{0}} \frac{p(\rho)-p\left(\rho_{0}\right)}{\rho-\rho_{0}}}
\end{array}\right.
$$

A discontinuity is called a shock if it satisfies the entropy condition (see [La]):

$$
\sigma\left(\eta(v)-\eta\left(v_{0}\right)\right)-\left(q(v)-q\left(v_{0}\right)\right) \geqq 0,
$$

for any convex entropy pair $(\eta, q)$.

There are two distinct types of rarefaction waves and shock waves denoted by $1-\mathrm{Rw}$ or $2-\mathrm{Rw}$ and 1 -shock or 2-shock, respectively, in the isentropic gases. If a state $\left(\rho_{0}, m_{0}\right)$ or $\left(\rho_{0}, u_{0}\right)$ is given, the possible states $(\rho, m)$ or $(\rho, u)$ that can be connected to $\left(\rho_{0}, m_{0}\right)$ on the right by a $\mathrm{Rw}$ or shock are

$$
\begin{aligned}
& R_{1}(0): m-m_{0}=\frac{m_{0}}{\rho_{0}}\left(\rho-\rho_{0}\right)-\frac{\rho}{\theta}\left(\rho^{\theta}-\rho_{0}^{\theta}\right), \quad \rho<\rho_{0}, \\
& R_{2}(0): m-m_{0}=\frac{m_{0}}{\rho_{0}}\left(\rho-\rho_{0}\right)+\frac{\rho}{\theta}\left(\rho^{\theta}-\rho_{0}^{\theta}\right), \quad \rho>\rho_{0}, \\
& S_{1}(0): m-m_{0}=\frac{m_{0}}{\rho_{0}}\left(\rho-\rho_{0}\right)-\sqrt{\frac{\rho}{\rho_{0}} \frac{p(\rho)-p\left(\rho_{0}\right)}{\rho-\rho_{0}}}\left(\rho-\rho_{0}\right), \quad \rho>\rho_{0}>0, \\
& S_{2}(0): m-m_{0}=\frac{m_{0}}{\rho_{0}}\left(\rho-\rho_{0}\right)+\sqrt{\frac{\rho}{\rho_{0}} \frac{p(\rho)-p\left(\rho_{0}\right)}{\rho-\rho_{0}}}\left(\rho-\rho_{0}\right), \quad \rho<\rho_{0},
\end{aligned}
$$

respectively.

Along the curve $R_{1}(0)$,

$$
\left.\frac{d m}{d \rho}\right|_{R_{1}(0)}=\frac{m_{0}}{\rho_{0}}+\frac{\rho_{0}^{\theta}}{\theta}-\frac{\theta+1}{\theta} \rho^{\theta},\left.\quad \frac{d^{2} m}{d \rho^{2}}\right|_{R_{1}(0)}=-(\theta+1) \rho^{\theta-1} \leqq 0,
$$

and along the curve $R_{2}(0)$,

$$
\left.\frac{d m}{d \rho}\right|_{R_{2}(0)}=\frac{m_{0}}{\rho_{0}}-\frac{\rho_{0}^{\theta}}{\theta}+\frac{\theta+1}{\theta} \rho^{\theta},\left.\quad \frac{d^{2} m}{d \rho^{2}}\right|_{R_{2}(0)}=(\theta+1) \rho^{\theta-1} \geqq 0 .
$$

This shows that the curve $R_{1}(0)$ is concave and the curve $R_{2}(0)$ is convex in the $\rho-m$ plane. 
Along the curve $S_{1}(0)$,

$$
\left\{\begin{array}{l}
\left.\frac{m-m_{0}}{\rho-\rho_{0}}\right|_{S_{1}(0)}=\frac{m_{0}}{\rho_{0}}-\sqrt{\frac{\rho}{\rho_{0}} \frac{p(\rho)-p\left(\rho_{0}\right)}{\rho-\rho_{0}}}, \\
\left.\frac{d}{d \rho}\left(\frac{m-m_{0}}{\rho-\rho_{0}}\right)\right|_{S_{1}(0)}=-\frac{\frac{\rho}{\rho_{0}} p^{\prime}(\rho)-\frac{p(\rho)-p\left(\rho_{0}\right)}{\rho-\rho_{0}}}{2 \sqrt{\frac{\rho}{\rho_{0}}\left(p(\rho)-p\left(\rho_{0}\right)\right)\left(\rho-\rho_{0}\right)}} \leqq 0, \quad \rho>\rho_{0}>0,
\end{array}\right.
$$

and along the curve $S_{2}(0)$,

$$
\left\{\begin{array}{l}
\left.\frac{m-m_{0}}{\rho-\rho_{0}}\right|_{S_{2}(0)}=\frac{m_{0}}{\rho_{0}}+\sqrt{\frac{\rho}{\rho_{0}} \frac{p(\rho)-p\left(\rho_{0}\right)}{\rho-\rho_{0}}}, \\
\left.\frac{d}{d \rho}\left(\frac{m-m_{0}}{\rho-\rho_{0}}\right)\right|_{S_{2}(0)}=-\frac{\frac{\rho}{\rho_{0}} p^{\prime}(\rho)-\frac{p(\rho)-p\left(\rho_{0}\right)}{\rho-\rho_{0}}}{2 \sqrt{\frac{\rho}{\rho_{0}}\left(p(\rho)-p\left(\rho_{0}\right)\right)\left(\rho-\rho_{0}\right)}} \geqq 0, \quad \rho<\rho_{0} .
\end{array}\right.
$$

This shows that the curve $S_{1}(0)$ is concave and the curve $S_{2}(0)$ is convex with respect to $\left(\rho_{0}, m_{0}\right)$ in the $\rho-m$ plane.

One wave is of particular interest, namely, the standing shock with the speed $\sigma=0$. For this case, the Rankine-Hugoniot conditions are

$$
m=m_{0}, \quad \frac{m^{2}}{\rho}+p=\frac{m_{0}^{2}}{\rho_{0}}+p\left(\rho_{0}\right) .
$$

Equations (2.6) can be rewritten as

$$
\frac{M}{M_{0}}=t^{-(\theta+1)}, \quad M_{0}^{2}=\frac{t\left(t^{\gamma}-1\right)}{\gamma(t-1)}=1+(\theta+1)(t-1)+\mathbf{O}\left(|t-1|^{2}\right)
$$

where $t=\rho / \rho_{0}$.

From (2.7), one has

$$
M^{2}=\frac{t^{\gamma}-1}{\gamma t^{\gamma}(t-1)}=1-(\theta+1)(t-1)+\mathbf{O}\left(|t-1|^{2}\right) .
$$

One can easily check that $M_{0}^{2}$ is a monotonically increasing function of $t$ with $M_{0}^{2}(0)=0, M_{0}^{2}(1)=1$, and $M_{0}^{2}(\infty)=\infty$; and $M^{2}$ is monotonically decreasing function of $t$ with $M^{2}(0)=\infty, M^{2}(1)=1$, and $M^{2}(\infty)=0$.

The entropy condition (2.5) for the standing shock (2.6) or (2.7) is

$$
\begin{cases}M<1<M_{0}, & \text { when } m_{0}>0, \\ M<-1<M_{0}, & \text { when } m_{0}<0 .\end{cases}
$$

2.2. Riemann Solutions. Similarly, given a state $\left(\rho_{0}, m_{0}\right)$ or $\left(\rho_{0}, u_{0}\right)$ for $\rho_{0}>0$, the locus of possible states $(\rho, m)$ or $(\rho, u)$ for $\rho>0$ that can be connected to the state on the left by a shock wave $S^{-1}$ or rarefaction wave $R^{-1}$ defines what is called an inverse shock wave curve or inverse rarefaction wave curve. It has behavior similar to that of $S$ or $R$.

From the behavior of these curves in the phase plane $(\rho, m)$, we can construct the unique solution for the Riemann problem

$$
\left.v\right|_{t=0}= \begin{cases}v_{-}, & x<x_{0} \\ v_{+}, & x>x_{0}\end{cases}
$$


and the Riemann initial-boundary problem

$$
\left.v\right|_{t=0}=v_{+},\left.\quad m\right|_{x=1}=0 .
$$

For the problem (2.8), we can get a diagram of the first family of elementary wave curves for given left state $v_{-}$and a diagram of the second family of inverse elementary wave curves for given right state $v_{+}$to determine a unique intersection point to obtain the unique solution. For the problem (2.9), we can draw a diagram of the second family of inverse elementary wave curves for given right state $v_{+}$ to determine a unique intersection point with the line $m=0$ to obtain the unique solution.

Theorem 2.1. There exists a unique piecewise smooth entropy solution $(\rho(x, t)$, $m(x, t))$ containing the vacuum state $(\rho=0)$ on the upper plane $t>0$ for each problem of (2.8) and (2.9) satisfying

(1) For the Riemann problem (2.8),

$$
\left\{\begin{array}{l}
w(\rho(x, t), m(x, t)) \leqq \max \left(w\left(\rho_{-}, m_{-}\right), w\left(\rho_{+}, m_{+}\right)\right), \\
z(\rho(x, t), m(x, t)) \geqq \min \left(z\left(\rho_{-}, m_{-}\right), z\left(\rho_{+}, m_{+}\right)\right), \\
w(\rho(x, t), m(x, t))-z(\rho(x, t), m(x, t)) \geqq 0 ;
\end{array}\right.
$$

(2) For the Riemann problem (2.9),

$$
\left\{\begin{array}{l}
w(\rho(x, t), m(x, t)) \leqq \max \left(w\left(\rho_{+}, m_{+}\right),-z\left(\rho_{+}, m_{+}\right)\right), \\
z(\rho(x, t), m(x, t)) \geqq \min \left(z\left(\rho_{+}, m_{+}\right), 0\right), \\
w(\rho(x, t), m(x, t))-z(\rho(x, t), m(x, t)) \geqq 0 .
\end{array}\right.
$$

Such Riemann solutions have the following properties:

Lemma 2.1. The regions $\sum=\left\{(\rho, m): w \leqq w_{0}, z \geqq z_{0}, w-z \geqq 0\right\}$ are invariant with respect to both of the Riemann problem (2.8) and the average of the Riemann solutions in $x$. More precisely, if the Riemann data lie in $\sum$, the corresponding Riemann solutions $(\rho(x, t), m(x, t))$ lie in $\sum$, and their corresponding averages in $x$ also lie in $\sum$ :

$$
\left(\frac{1}{b-a} \int_{a}^{b} \rho(x, t) d x, \frac{1}{b-a} \int_{a}^{b} m(x, t) d x\right) \in \sum .
$$

Furthermore, for the Riemann initial-boundary problem (2.9), the regions $\sum=$ $\left\{(\rho, m): w \leqq w_{0}, z \geqq z_{0}, w-z \geqq 0\right\}, z_{0} \leqq 0 \leqq \frac{w_{0}+z_{0}}{2}$, are invariant with respect to both of the Riemann problem (2.9) and the average of the corresponding Riemann solutions in $x$.

The proof of Lemma 2.1 can be found in [C1, MT].

Lemma 2.2. The rate of entropy production of a shock with left state $v_{-}$and right state $v_{+}$for an arbitrary weak entropy $\eta$ is dominated by the associated rate of entropy production for $\eta_{*}$ in the following sense:

$$
\begin{aligned}
& \left|\sigma\left(\eta\left(v_{+}\right)-\eta\left(v_{-}\right)\right)-\left(q\left(v_{+}\right)-q\left(v_{-}\right)\right)\right| \\
& \quad \leqq C\left\{\sigma\left(\eta_{*}\left(v_{+}\right)-\eta_{*}\left(v_{-}\right)\right)-\left(q_{*}\left(v_{+}\right)-q_{*}\left(v_{-}\right)\right)\right\},
\end{aligned}
$$

where the constant $C$ depends only on $\eta$ and $\max \left(\left|\rho_{ \pm}\right|+\left|\frac{m_{ \pm}}{\rho_{ \pm}}\right|\right)$.

The proof of this fact can be found in [C1]. 


\section{Steady-State Solutions}

Travelling waves and Riemann solutions of the homogeneous problems (2.8) and (2.9) have been discussed in Sect. 2. The purpose of this section is to provide important estimates on steady-state solutions of the inhomogeneous problem (1.2) determined by the following system of ordinary differential equations:

$$
\left\{\begin{array}{l}
m_{x}=-\frac{A^{\prime}(x)}{A(x)} m \\
\left(\frac{m^{2}}{\rho}+p(\rho)\right)_{x}=-\frac{A^{\prime}(x)}{A(x)} \frac{m^{2}}{\rho}
\end{array}\right.
$$

subject to the boundary condition

$$
\left.(\rho, m)\right|_{x=x_{0}}=\left(\rho_{0}, m_{0}\right) .
$$

The nonsonic and transonic cases are distinct, as the former produces smooth solutions and the latter may contain a standing shock wave. The $L^{\infty}$ estimates are derived based on Riemann invariant inequalities and are required for the compensated compactness framework. The $L^{1}$ estimates are needed for consistency and verification of the entropy condition.

In this section we always assume that $A(x)$ is a $C^{2}$ function satisfying $A(x) \geqq$ $c_{0}>0$ in the interval under consideration.

3.1. Smooth Steady-State Solutions for the Nonsonic Case. We first consider the nonsonic case $M_{0}^{2} \approx 1$, where $M_{0}=M\left(x=x_{0}\right)=\frac{m_{0}}{\rho_{0} c_{0}}$.

The first equation can be directly integrated to obtain

$$
A(x) m=A\left(x_{0}\right) m_{0} .
$$

The second equation can be rewritten as

$$
\left(A(x) \frac{m^{2}}{\rho}\right)_{x}+A(x) p(\rho)_{x}=0,
$$

and, using (3.3),

$$
m\left(\frac{m}{\rho}\right)_{x}+p(\rho)_{x}=0
$$

that is,

$$
\left(\frac{m^{2}}{2 \rho^{2}}\right)_{x}+\left(\int_{0}^{\rho} \frac{p^{\prime}(s)}{s} d s\right)_{x}=0 .
$$

Therefore, one has

$$
\frac{u^{2}}{2}+\int_{0}^{\rho} \frac{p^{\prime}(s)}{s} d s=\frac{u_{0}}{2}+\int_{0}^{\rho_{0}} \frac{p^{\prime}(s)}{s} d s .
$$

In terms of $\rho, M$, and $M_{0}$, the system (3.3)-(3.4) becomes

$$
\begin{aligned}
& \frac{A(x) M}{A\left(x_{0}\right) M_{0}}=\left(\frac{\rho_{0}}{\rho}\right)^{\theta+1}, \\
& \left(\frac{\theta M^{2}+1}{\theta M_{0}^{2}+1}\right)^{\frac{1}{2 \theta}}=\frac{\rho_{0}}{\rho} .
\end{aligned}
$$


Eliminating the variable $\rho$ in $(3.5)-(3.6)$, one has

$$
\frac{M}{\left(1+\theta M^{2}\right)^{\frac{\theta+1}{2 \theta}}} A(x)=\frac{M_{0}}{\left(1+\theta M_{0}^{2}\right)^{\frac{\theta+1}{2 \theta}}} A\left(x_{0}\right) .
$$

The solution of the steady-state equations is thus reduced to the following procedure: solve Eq. (3.7) for $M$ and use (3.5) or (3.6) to obtain $\rho$.

Equation (3.7) can be rewritten as

$$
F(M)=\frac{A\left(x_{0}\right)}{A(x)} F\left(M_{0}\right),
$$

where the function $F$ is defined by

$$
F(M)=M\left(\frac{1+\theta}{1+\theta M^{2}}\right)^{\frac{\theta+1}{2 \theta}}
$$

satisfying

$$
\begin{cases}F(0)=0, F(1)=1, F(M) \rightarrow 0, & \text { when } M \rightarrow \infty, \\ F^{\prime}(M)(1-M)>0, & \text { when } M \in[0, \infty), \\ F^{\prime}(M)(1+M)>0, & \text { when } M \in(-\infty, 0] .\end{cases}
$$

Thus we see that there are two difficulties in solving function equation (3.8): If $A(x)<A\left(x_{0}\right)\left|F\left(M_{0}\right)\right|$, no smooth solution exists, since the right side of (3.8) exceeds the maximum value of $|F|$. If $A(x)>A\left(x_{0}\right)\left|F\left(M_{0}\right)\right|$, there are two solutions of (3.8), one with $|M|>1$ and the other with $|M|<1$ since the line $F=$ const. intersects the graph of $F$ at two points. As long as $|M| \neq 1$ is maintained, exactly one of these solutions is smooth for the problem (3.1)-(3.2).

Lemma 3.1. Let $v(x)$ be a smooth steady-state solution satisfying $\left.v\right|_{x=x_{0}}=v_{0}$, with $\rho_{0} \geqq 0$, in an interval $[a, b]$ containing $x_{0}$. Then

$$
\rho(x) \geqq 0, \quad x \in[a, b] .
$$

The next two lemmas will be used in deriving $L^{\infty}$ estimates. The main idea is that the quadrant in the Riemann invariant plane, which is invariant for the homogeneous hyperbolic equations, is approximately invariant for the steady inhomogeneous equations. Let $M=M(v(x))$ and $M_{0}=M\left(v_{0}\right)$ be the Mach numbers. An important intermediate step is to establish Lipschitz or Hölder continuity of a relative Mach number $\frac{M}{M_{0}}$.

Lemma 3.2. Let $v(x)$ be the smooth steady-state solution satisfying $\left.v\right|_{x=x_{0}}=v_{0}$. Then, given a sufficiently small $\varepsilon_{0} \in\left(0, \frac{1}{2}\right)$, there exists $h_{1}=h_{1}\left(\varepsilon_{0}\right) \in(0,1]$ and $C>0$ depending only on the function $A(x)$ such that, in any interval $\left[x_{0}-\frac{h}{2}, x_{0}+\right.$ $\left.\frac{h}{2}\right] \subset[a, b], h \leqq h_{1}$,

(i) when $\left|M_{0}^{2}-1\right| \geqq \varepsilon_{0} M_{0}^{2}$,

$$
\left|\frac{M-M_{0}}{M_{0}}\right| \leqq C\left|x-x_{0}\right|,
$$


(ii) when $h^{\beta} M_{0}^{2} \leqq\left|M_{0}^{2}-1\right|<\varepsilon_{0} M_{0}^{2}$ with some $\beta \in\left(0, \frac{1}{6}\right)$,

$$
\left\{\begin{array}{l}
\left|\frac{M-M_{0}}{M_{0}}\right|\left|\frac{1-\left(M_{0}+\tau_{0}\left(M-M_{0}\right)\right)^{2}}{1+\left(M_{0}+\tau_{0}\left(M-M_{0}\right)\right)^{2}}\right| \leqq C\left|x-x_{0}\right|, \text { for some } \tau_{0} \in(0,1), \\
\left|\frac{M-M_{0}}{M_{0}}\right| \leqq C \sqrt{\left|x-x_{0}\right|}
\end{array}\right.
$$

Proof. We consider separately these two cases. Before introducing this distinction, we recast the relative geometrical factor $A\left(x_{0}\right) / A(x)$ in terms of the steady-state Mach number $N=M / M_{0}$. By (3.7)

$$
\frac{A\left(x_{0}\right)}{A(x)}=\frac{M}{M_{0}}\left(\frac{1+\theta M_{0}^{2}}{1+\theta M^{2}}\right)^{\frac{\theta+1}{2 \theta}}
$$

Define

$$
G\left(N ; M_{0}\right)=N\left(\frac{1+\theta M_{0}^{2}}{1+\theta M_{0}^{2} N^{2}}\right)^{\frac{\theta+1}{2 \theta}}
$$

Then $N=N(x)$ satisfies

$$
\left\{\begin{array}{l}
G\left(N ; M_{0}\right)=\frac{A\left(x_{0}\right)}{A(x)}=1+\frac{A\left(x_{0}\right)-A(x)}{A(x)} \\
\left.N\right|_{x=x_{0}}=1
\end{array}\right.
$$

Our purpose is to control $N(x)-1$ by $\left|x-x_{0}\right|$. For the case $\left|M_{0}^{2}-1\right| \geqq$ $\varepsilon_{0} M_{0}^{2}, G\left(N ; M_{0}\right)$ is uniformly monotone near $N=1$ with respect to $M_{0}$, and so (3.9) provides an upper bound on $N-1$ as we now establish. In this case,

$$
M_{0}^{2} \leqq \frac{1}{1+\varepsilon_{0}} \quad \text { or } \quad M_{0}^{2} \geqq \frac{1}{1-\varepsilon_{0}} .
$$

Notice that, in the interval $N \in\left[\sqrt{1-\frac{\varepsilon_{0}}{2}}, \sqrt{1+\frac{\varepsilon_{0}}{2}}\right]$, there exists $c\left(\varepsilon_{0}\right)>0$, independent of $M_{0}$, such that

$$
\left|G^{\prime}\left(N ; M_{0}\right)\right|=\left(\frac{1+\theta M_{0}^{2}}{1+\theta M_{0}^{2} N^{2}}\right)^{\frac{\theta+1}{2 \theta}} \frac{\left|1-M_{0}^{2} N^{2}\right|}{1+\theta M_{0}^{2} N^{2}} \geqq c\left(\varepsilon_{0}\right)>0,
$$

which means that $G\left(N ; M_{0}\right)$ is uniformly monotone in $N \in\left[\sqrt{1-\frac{\varepsilon_{0}}{2}}, \sqrt{1+\frac{\varepsilon_{0}}{2}}\right]$. Therefore, we obtain that there exist $\tilde{h}\left(\varepsilon_{0}\right)>0$ and $C_{1}\left(\varepsilon_{0}\right)>0$, independent of $M_{0}$, such that, whenever $\left|x-x_{0}\right| \leqq \frac{\tilde{h}}{2}$,

$$
|N(x)-1| \leqq C_{1}\left(\varepsilon_{0}\right)\left|x-x_{0}\right|,
$$

using (3.10).

We now consider the case $h^{\beta} M_{0}^{2} \leqq\left|M_{0}^{2}-1\right|<\varepsilon_{0} M_{0}^{2}$, that is,

$$
\frac{1}{1+\varepsilon_{0}}<M_{0}^{2} \leqq \frac{1}{1+h^{\beta}}, \quad \text { or } \frac{1}{1-h^{\beta}} \leqq M_{0}^{2}<\frac{1}{1-\varepsilon_{0}},
$$

in which (3.9) will provide an upper bound on $(N(x)-1)^{2}$. 
For this case, in the interval $N \in\left[\sqrt{1-\frac{\varepsilon_{0}}{2}}, \sqrt{1+\frac{\varepsilon_{0}}{2}}\right], G^{\prime}\left(N ; M_{0}\right)$ changes sign at most once. Moreover, there exists $\alpha\left(\varepsilon_{0}\right)>0$, independent of $M_{0}$, such that

$$
G^{\prime \prime}\left(N ; M_{0}\right)=-(\theta+1)\left(\frac{1+\theta M_{0}^{2}}{1+\theta M_{0}^{2} N^{2}}\right)^{\frac{\theta+1}{2 \theta}} \frac{M_{0}^{2} N\left(3-M_{0}^{2} N^{2}\right)}{\left(1+\theta M_{0}^{2} N^{2}\right)^{2}} \leqq-\alpha\left(\varepsilon_{0}\right) .
$$

Therefore, there exists $\tilde{h}_{1}\left(\varepsilon_{0}\right) \leqq \tilde{h}\left(\varepsilon_{0}\right)<1$, independent of $M_{0}>0$, such that, when $\left|x-x_{0}\right| \leqq \frac{\tilde{h}_{1}\left(\varepsilon_{0}\right)}{2}, N(x)$ satisfies

$$
|N(x)-1| \leqq \frac{\varepsilon_{0}}{2}
$$

from (3.9).

From (3.9) and the Mean Value Theorem, there exists $\tau_{0} \in(0,1)$ such that

$$
G^{\prime}\left(1+\tau_{0}(N-1) ; M_{0}\right)(N-1)=\frac{A\left(x_{0}\right)-A(x)}{A(x)} .
$$

This implies that, when $\left|x-x_{0}\right| \leqq \frac{\tilde{h}_{1}}{2}$,

$$
\begin{aligned}
& \left|\frac{N-1}{x-x_{0}}\right|\left|\frac{1-M_{0}^{2}\left(1+\tau_{0}(N-1)\right)^{2}}{1+\theta M_{0}^{2}\left(1+\tau_{0}(N-1)\right)^{2}}\right| \\
& \quad \leqq\left|\frac{A\left(x_{0}\right)-A(x)}{A(x)\left(x-x_{0}\right)}\right|\left(\frac{1+\theta M_{0}^{2}\left(1+\tau_{0}(N-1)\right)^{2}}{1+\theta M_{0}^{2}}\right)^{\frac{\theta+1}{2 \theta}} \leqq C_{2}\left(\varepsilon_{0}\right),
\end{aligned}
$$

using the formula of $G^{\prime}\left(N ; M_{0}\right)$.

Furthermore, using the Mean Value Theorem, there exists $\tilde{\tau} \in(0,1)$ such that

$$
a(\tilde{\tau}, N)(N-1)^{2}+b\left(M_{0}\right)(N-1)+c\left(x, x_{0}\right)=0,
$$

where

$$
\left\{\begin{array}{l}
a(\tilde{\tau}, N)=-\frac{1}{2} G^{\prime \prime}(1+\tilde{\tau}(N-1))>0, \\
b\left(M_{0}\right)=\frac{M_{0}^{2}-1}{1+\theta M_{0}^{2}}, \quad c\left(x, x_{0}\right)=\frac{A\left(x_{0}\right)-A(x)}{A(x)} .
\end{array}\right.
$$

Then there exists $h_{1}\left(\varepsilon_{0}\right) \leqq \tilde{h}_{1}\left(\varepsilon_{0}\right)$ such that, when $h \leqq h_{1}\left(\varepsilon_{0}\right)$,

$$
h^{\beta} \geqq 3 \sqrt{h} ; \quad b\left(M_{0}\right)^{2}-4 a(\tilde{\tau}, N) c\left(x, x_{0}\right)>0, \quad \text { for }\left|x-x_{0}\right|<h .
$$

Therefore,

$$
|N(x)-1|=\frac{2\left|x-x_{0}\right|}{\left|b\left(M_{0}\right)\right|+\sqrt{b\left(M_{0}\right)^{2}-4 a(\tilde{\tau}, N) c\left(x, x_{0}\right)}} \leqq C_{2}\left(\varepsilon_{0}\right) \sqrt{\left|x-x_{0}\right|},
$$

when $\left|x-x_{0}\right| \leqq \frac{h}{2}, h \leqq h_{1}$, for the case $\left|M_{0}^{2}-1\right| \leqq \varepsilon_{0} M_{0}^{2}$ by using $\left|b\left(M_{0}\right)\right| \geqq$ $\frac{1}{3} h^{\beta} \geqq \sqrt{h}$. Choosing $C\left(\varepsilon_{0}\right)=\max _{i=1,2} C_{i}\left(\varepsilon_{0}\right)$, we prove the lemma. 
We now estimate the Riemann invariants in order to derive $L^{\infty}$ bounds on the approximate solutions.

Lemma 3.3. Let $v(x)$ be a smooth steady-state solution satisfying $\left.v\right|_{x=x_{0}}=v_{0}$ in $\left[x_{0}-\frac{h}{2}, x_{0}+\frac{h}{2}\right], h \leqq h_{1}$, with $\left|M_{0}^{2}-1\right| \geqq h^{\beta} M_{0}^{2}$. Then, when $\left|x-x_{0}\right| \leqq \frac{h}{2}$, we have

$$
\begin{cases}w(v(x)) \leqq w\left(v_{0}\right)\left(1+C\left|x-x_{0}\right|\right), & \text { when } M_{0}>0, \\ z(v(x)) \geqq z\left(v_{0}\right)\left(1+C\left|x-x_{0}\right|\right), & \text { when } M_{0}<0,\end{cases}
$$

where $(w, z)$ are the Riemann invariants, $h_{1}>0$ is the constant determined in Lemma 3.2, and $C$ is a constant depending only on the function $A(x)$.

Proof. We divide the case $M_{0}>0$ into two subcases. In the case $\left|M_{0}^{2}-1\right| \geqq \varepsilon_{0} M_{0}^{2}$, we use the estimate for $N-1$ in Lemma 3.2 , when $\left|x-x_{0}\right| \leqq \frac{h}{2}, h \leqq h_{1}$, to obtain

$$
\begin{aligned}
\left(\frac{w(v)}{w\left(v_{0}\right)}\right)^{2} & =1+\frac{\theta M_{0}\left[\left(1-M_{0}\right)\left(1+\theta M_{0} N\right)+\left(1-M_{0} N\right)\left(1+\theta M_{0}\right)\right] \frac{N-1}{x-x_{0}}}{\left(\theta M_{0}+1\right)^{2}\left(1+\theta M_{0}^{2} N^{2}\right)}\left(x-x_{0}\right) \\
& \leqq 1+C\left|x-x_{0}\right| .
\end{aligned}
$$

In the case $h^{\beta} M_{0}^{2} \leqq\left|M_{0}^{2}-1\right| \leqq \varepsilon_{0} M_{0}^{2}$, we have

$$
\begin{aligned}
\left(\frac{w(v)}{w\left(v_{0}\right)}\right)^{2} & =1+\frac{\theta M_{0}\left\{\begin{array}{l}
2\left(1+\theta M_{0}\right)\left[1-M_{0}\left(1+\tau_{0}(N-1)\right)\right] \frac{N-1}{x-x_{0}} \\
+M_{0}\left[\theta\left(1-M_{0}\right)+\left(2 \tau_{0}-1\right)\left(1+\theta M_{0}\right)\right] \frac{(N-1)^{2}}{x-x_{0}}
\end{array}\right\}}{\left(\theta M_{0}+1\right)^{2}\left(1+\theta M_{0}^{2} N^{2}\right)}\left(x-x_{0}\right) \\
& \leqq 1+C\left|x-x_{0}\right|,
\end{aligned}
$$

when $\left|x-x_{0}\right| \leqq \frac{h}{2}$ and $\tau_{0} \in(0,1)$ is the same as the one in (3.11)-(3.12), using the estimates (3.12) and (3.13) for $N-1$ in the transonic case by noting

$$
1 \leqq 1+\theta M_{0}^{2}\left(1+\tau_{0}(N-1)\right)^{2} \leqq 1+\left(1+C h_{1}\right)^{2} \leqq C .
$$

Similarly, for the case $M_{0}<0$, we have

$$
\begin{aligned}
\left(\frac{z(v)}{z\left(v_{0}\right)}\right)^{2} & =1-\frac{\theta M_{0}\left[\left(1+M_{0}\right)\left(1-\theta M_{0} N\right)+\left(1-\theta M_{0}\right)\left(1+M_{0} N\right)\right] \frac{N-1}{x-x_{0}}}{\left(1-\theta M_{0}\right)^{2}\left(1+\theta M_{0}^{2} N^{2}\right)}\left(x-x_{0}\right) \\
& \leqq 1+C\left|x-x_{0}\right|,
\end{aligned}
$$

by using Lemma 3.2. The estimate (3.14) follows.

Now we derive $L^{1}$ estimates on the difference between the smooth steady-state solution over $\left[x_{0}-\frac{h}{2}, x_{0}+\frac{h}{2}\right]$ and the boundary data at $x_{0}$ for sufficiently small $h$. We consider here the nonsonic case $\left|M_{0}^{2}-1\right| \geqq h^{\beta} M_{0}^{2}$ for some $\beta \in\left(0, \frac{1}{6}\right]$.

Lemma 3.4. There exist $h_{2}>0,0<h_{2} \leqq h_{1}$, and a smooth steady-state solution in $\left[x_{0}-\frac{h}{2}, x_{0}+\frac{h}{2}\right], h \leqq h_{2}$, with boundary condition $\left.v\right|_{x=x_{0}}=v_{0}$ such that, when $\left|M_{0}^{2}-1\right| \geqq h^{\beta} M_{0}^{2}$,

$$
v(x)=v_{0}\left(1+\mathbf{O}\left(h^{1-\beta}\right)\right), \quad \frac{1}{h} \int_{x_{0}-\frac{h}{2}}^{x_{0}+\frac{h}{2}} v(x) d x=v_{0}\left(1+\mathbf{O}\left(h^{2(1-\beta)}\right)\right),
$$


where bounds $\mathbf{O}\left(h^{1-\beta}\right)$ and $\mathbf{O}\left(h^{2(1-\beta)}\right)$ depend only on bounds of $A(x)$ and $\rho_{0}+$ $\left|\frac{m_{0}}{\rho_{0}}\right|$ and are independent of $M_{0}$.

Proof. The estimates we expect are for averaging the smooth steady-state solution over the symmetric interval $\left[x_{0}-\frac{h}{2}, x_{0}+\frac{h}{2}\right]$. The basic idea of our proof is to take Taylor's expansion for the solution up to the second order as the reminder. The point is that the coefficient in the first order term is constant and the averaging integral cancels this term. Therefore, the second order reminder term is the first correction for the averaging integral and we only need to estimate the second derivative of the solution.

With this strategy in mind, we first use (3.3) and $A(x) \in C^{2}\left[x_{0}-\frac{h}{2}, x_{0}+\frac{h}{2}\right]$, and take Taylor's expansion for $m(x)$ at $x=x_{0}$ :

$$
m(x)=m_{0} \frac{A\left(x_{0}\right)}{A(x)}=m_{0}\left(1-\frac{A^{\prime}\left(x_{0}\right)}{A\left(x_{0}\right)}\left(x-x_{0}\right)+\frac{1}{2} A\left(x_{0}\right)\left(\frac{1}{A}\right)^{\prime \prime}(\tilde{y})\left(x-x_{0}\right)^{2}\right),
$$

for some $\tilde{y} \in\left(x_{0}-\frac{h}{2}, x_{0}+\frac{h}{2}\right)$. We conclude

$$
\frac{1}{h} \int_{x_{0}-\frac{h}{2}}^{x_{0}+\frac{h}{2}} m(x) d x=m_{0}+A\left(x_{0}\right) m_{0} \frac{1}{2 h} \int_{x_{0}-\frac{h}{2}}^{x_{0}+\frac{h}{2}}\left(\frac{1}{A}\right)^{\prime \prime}(\tilde{y})\left(x-x_{0}\right)^{2} d x=m_{0}\left(1+\mathbf{O}\left(h^{2}\right)\right) \text {. }
$$

Similarly, we expand $\rho(x)$ at $x=x_{0}$ :

$$
\rho(x)=\rho_{0}+\rho^{\prime}\left(x_{0}\right)\left(x-x_{0}\right)+\frac{1}{2} \rho^{\prime \prime}(y)\left(x-x_{0}\right)^{2},
$$

for some $y \in\left(x_{0}-\frac{h}{2}, x_{0}+\frac{h}{2}\right)$ and only need to estimate $\rho^{\prime \prime}(y)$.

A careful calculation leads to

$$
\rho^{\prime}(y)=\rho_{0} \frac{A^{\prime}(y)}{A(y)}\left(\frac{\theta M_{0}^{2}+1}{\theta M^{2}+1}\right)^{\frac{1}{2 \theta}} \frac{M^{2}}{1-M^{2}}, \quad \rho^{\prime \prime}(y)=\rho_{0} \frac{\left(\theta M_{0}^{2}+1\right)^{\frac{1}{2 \theta}}}{\left(\theta M^{2}(y)+1\right)^{\frac{4 \theta+1}{2 \theta}}} D(y),
$$

where

$$
\begin{aligned}
D(y)= & {\left[(\theta+1) M^{2}(y)-1\right]\left(M^{\prime}(y)\right)^{2}-M(y)\left(\theta M^{2}(y)+1\right) M^{\prime \prime}(y) } \\
= & \left(A\left(x_{0}\right) \frac{A^{\prime}(y)}{A^{2}(y)}\right)^{2} \frac{\left((\theta+1) M^{2}(y)-1\right) F^{2}\left(M_{0}\right)}{\left(F^{\prime}(M(y))\right)^{2}} \\
& -\frac{A\left(x_{0}\right)}{A^{4}(y)} \frac{M(y)\left(1+\theta M^{2}(y)\right) F\left(M_{0}\right)}{\left(F^{\prime}(M(y))\right)^{3}} \\
& \times\left(\left(2\left(A^{\prime}(y)\right)^{2}-A(y) A^{\prime \prime}(y)\right) A(y)\left(F^{\prime}(M(y))\right)^{2}\right. \\
& \left.-A\left(x_{0}\right)\left(A^{\prime}(y)\right)^{2} F\left(M_{0}\right) F^{\prime \prime}(M(y))\right) .
\end{aligned}
$$


We obtain that there exists $\hat{h} \leqq h_{1}$ such that, when $\left|y-x_{0}\right| \leqq \frac{h}{2}, h \leqq \hat{h}$,

$$
\left|\rho^{\prime}(y)\right| \leqq \begin{cases}C \rho_{0} \frac{M_{0}^{2}}{1-M_{0}^{2}}, & M_{0}^{2} \geqq \frac{1}{2} \\ C \rho_{0}, & M_{0}^{2}<\frac{1}{2}\end{cases}
$$

and

$$
\left|\rho^{\prime \prime}(y)\right| \leqq\left\{\begin{array}{l}
C \rho_{0} \frac{M_{0}^{4}}{\left(1-M^{2}(y)\right)^{2}}\left(1+\frac{M_{0}^{2}}{\left|1-M^{2}(y)\right|}\right), \quad M_{0}^{2} \geqq \frac{1}{2}, \\
C \rho_{0}, \quad M_{0}^{2}<\frac{1}{2},
\end{array}\right.
$$

where $C$ is a constant independent of $\rho_{0}$ and $M_{0}$.

The estimates (3.15)-(3.16), especially for the case $M_{0}^{2} \leqq \frac{1}{2}$, are obtained by using Lemma 3.2, which ensures that there exists $C>0$ such that, when $\left|y-x_{0}\right| \leqq$ $\frac{h}{2}, h \leqq \hat{h}$,

$$
\left|M(y)-M_{0}\right| \leqq C \sqrt{\left|y-x_{0}\right|}\left|M_{0}\right|
$$

For the case $M_{0}^{2} \geqq \frac{1}{2}$, we have

$$
\begin{aligned}
\left|1-M^{2}(y)\right| & \geqq\left|1-M_{0}^{2}\right|-\left|M(y)-M_{0}\right|\left|M(y)+M_{0}\right| \\
& \geqq M_{0}^{2}\left(h^{\beta}-C \sqrt{\left|y-x_{0}\right|}\left(1+C \sqrt{\left|y-x_{0}\right|}\right)\right) \geqq \frac{h^{\beta}}{2} M_{0}^{2},
\end{aligned}
$$

when $\left|y-x_{0}\right| \leqq \frac{h}{2}, h \leqq h_{2}$ for sufficiently small $h_{2} \leqq \hat{h}$.

This implies from (3.15)-(3.16) that

$$
\left|\rho^{\prime}(y)\right| \leqq C \rho_{0} h^{-\beta}, \quad\left|\rho^{\prime \prime}(y)\right| \leqq C \rho_{0} h^{-2 \beta} .
$$

We also conclude that, when $h \leqq h_{2}$,

$$
\rho(x)=\rho_{0}\left(1+\mathbf{O}\left(h^{1-\beta}\right)\right), \quad \frac{1}{h} \int_{x_{0}-\frac{h}{2}}^{x_{0}+\frac{h}{2}} \rho(x) d x=\rho_{0}\left(1+\mathbf{O}\left(h^{2(1-\beta)}\right)\right) .
$$

This completes the proof of Lemma 3.4.

3.2. Approximate Steady-State Solutions Near the Sonic State. The case $\left|M_{0}^{2}-1\right|$ $\leqq h^{\beta} M_{0}^{2}$ includes $A(x)<A\left(x_{0}\right) F\left(M_{0}\right)$, for which the solution must be discontinuous and a standing shock wave must be introduced at some point in $\left(x_{0}-\frac{h}{2}, x_{0}+\frac{h}{2}\right)$ in general. In this case we construct approximate steady-state solutions to make detailed estimates in a clear fashion.

Consider the problem (3.1)-(3.2) for the case $K_{0} \sqrt{h} \leqq\left|M_{0}^{2}-1\right| \leqq h^{\beta} M_{0}^{2}$ with

$$
K_{0}=2 \sqrt{\frac{\|a\|_{C}}{\theta}}
$$

where $K_{0}$ is chosen to ensure the following lemmas, specially Lemma 3.8 . 
Definition 3.1. The pair $(\rho, m)$ satisfies (3.1) in the approximate sense provided

$$
\left\{\begin{array}{l}
\left|m_{x}+a(x) m\right| \leqq \mathbf{o}(1) \\
\left|\left(\frac{m^{2}}{\rho}+\frac{\rho^{\gamma}}{\gamma}\right)_{x}+a(x) \frac{m^{2}}{\rho}\right| \leqq \mathbf{o}(1), \quad \text { as } h \rightarrow 0 .
\end{array}\right.
$$

Then we have the following approximate steady-state solutions.

Lemma 3.5. Let $a_{0}=a\left(x_{0}\right) \equiv \frac{A^{\prime}\left(x_{0}\right)}{A\left(x_{0}\right)}$. There exists $h_{3} \in(0,1]$ such that, for any interval $\left[x_{0}-\frac{h}{2}, x_{0}+\frac{h}{2}\right]$ with $h \leqq h_{3}$ and with $K_{0} \sqrt{h} \leqq\left|M_{0}^{2}-1\right| \leqq h^{\beta} M_{0}^{2}$, the functions

$$
\left\{\begin{aligned}
\rho(x)= & \rho_{0}\left(1+\frac{M_{0}^{2}-1}{2(\theta+1)}\left(1-\sqrt{1+\frac{4(\theta+1) a_{0}}{\left(M_{0}^{2}-1\right)^{2}}(x-\tilde{x})}\right)\right), \\
& \quad \text { for any } \tilde{x} \in\left(x_{0}-\frac{h}{2}, x_{0}+\frac{h}{2}\right) \\
n(x)= & m_{0}\left(1-a_{0}\left(x-x_{0}\right)\right),
\end{aligned}\right.
$$

satisfy (3.1) in the approximate sense and the bounds $\mathbf{0}(1)$ in Definition 3.1 depend only on $\rho_{0}+\left|\frac{m_{0}}{\rho_{0}}\right|$ and $a(x) \in C^{1}$.

Proof. Substituting the right side of (3.18) into the $m$ equation, one has

$$
\begin{aligned}
\left|m_{x}+a(x) m\right| & =\left|m_{0}\right|\left|\left(a(x)-a_{0}\right)-a_{0} a(x)\left(x-x_{0}\right)\right| \\
& \leqq\left|m_{0}\right|\left(\left\|a^{\prime}\right\|_{L^{\infty}}+\|a\|_{L^{\infty}}^{2}\right)\left|x-x_{0}\right| \leqq C h .
\end{aligned}
$$

Similarly, we have

$$
\begin{aligned}
& \left|\left(\frac{m^{2}}{\rho}+\frac{\rho^{\gamma}}{\gamma}\right)_{x}+a(x) \frac{m^{2}}{\rho}\right| \\
& \quad \leqq\left|\frac{m}{\rho}\right|\left|a(x) m_{0}\left(1-a_{0}\left(x-x_{0}\right)\right)-a_{0} m_{0}\right|+\left|\left(\rho^{2 \theta}-\frac{m^{2}}{\rho^{2}}\right) \rho_{x}-a_{0} \frac{m_{0} m}{\rho}\right| \\
& \quad \leqq\left|\frac{m}{\rho}\right|_{L^{\infty}}\left|m_{0}\right|\left(\left\|a^{\prime}\right\|_{L^{\infty}}+\left\|a^{2}\right\|_{L^{\infty}}\right)\left|x-x_{0}\right|+\Delta_{h},
\end{aligned}
$$

where

$$
\Delta_{h}=\left|\left(\rho^{2 \theta}-\frac{m^{2}}{\rho^{2}}\right) \rho_{x}-a_{0} \frac{m_{0} m}{\rho}\right| .
$$

We rewrite the $\rho$ equation in (3.18) as

$$
\rho(x)=\rho_{0}(1+P(x)), \quad P(x)=\frac{M_{0}^{2}-1}{2(\theta+1)}\left(1-\sqrt{1+\frac{4(\theta+1) a_{0}}{\left(M_{0}^{2}-1\right)^{2}}(x-\tilde{x})}\right) .
$$

From the formula of $P(x)$, there exists $h_{3}>0$ such that, when $h \leqq h_{3}$,

$$
|P(x)| \leqq\left|M_{0}^{2}-1\right| \leqq \frac{1}{2}, \quad \text { for } x \in\left[x_{0}-\frac{h}{2}, x_{0}+\frac{h}{2}\right] \text {. }
$$


Using this expression for $\rho$ and the $m$ equation in (3.18), we have

$$
\begin{aligned}
& \Delta_{h}=\frac{\rho_{0}^{2 \theta+1}\left|a_{0}\right|}{(1+P(x))^{2} \sqrt{1+\frac{4(\theta+1) a_{0}}{\left(M_{0}^{2}-1\right)^{2}}(x-\tilde{x})}} \\
& \times\left|\begin{array}{l}
\frac{1}{M_{0}^{2}-1}\left[(1+P(x))^{2 \theta+2}-M_{0}^{2}\left(1-a_{0}\left(x-x_{0}\right)\right)^{2}\right] \\
+M_{0}^{2}(1+P(x))\left(1-a_{0}\left(x-x_{0}\right)\right) \sqrt{1+\frac{4(\theta+1) a_{0}}{\left(M_{0}^{2}-1\right)^{2}}(x-\tilde{x})}
\end{array}\right| \\
& \leqq \frac{\rho_{0}^{2 \theta+1}\left|a_{0}\right|}{(1+P(x))^{2} \sqrt{1+\frac{4(\theta+1) a_{0}}{\left(M_{0}^{2}-1\right)^{2}}(x-\tilde{x})}} \\
& \times\left(\begin{array}{l}
\left|\frac{1}{M_{0}^{2}-1}\left(1-M_{0}^{2}+2(\theta+1) P(x)\right)+M_{0}^{2} \sqrt{1+\frac{4(\theta+1) a_{0}}{\left(M_{0}^{2}-1\right)^{2}}(x-\tilde{x})}\right| \\
+\mathbf{O}\left(\left|M_{0}^{2}-1\right|+\left|\frac{x-x_{0}}{M_{0}^{2}-1}\right|\right)
\end{array}\right) \\
& =\frac{\rho_{0}^{2 \theta+1}\left|a_{0}\right|}{(1+P(x))^{2}} \mathbf{O}\left(\left|M_{0}^{2}-1\right|+\left|\frac{x-x_{0}}{M_{0}^{2}-1}\right|\right) \text {. }
\end{aligned}
$$

We conclude that

$$
\Delta_{h} \leqq \mathbf{O}\left(\left|M_{0}^{2}-1\right|+\left|\frac{x-x_{0}}{M_{0}^{2}-1}\right|\right) \leqq \mathbf{O}\left(h^{\beta}\right)
$$

that is,

$$
\left|\left(\frac{m^{2}}{\rho}+\frac{\rho^{\gamma}}{\gamma}\right)_{x}+a(x) \frac{m^{2}}{\rho}\right| \leqq \mathbf{O}\left(h^{\beta}\right) .
$$

This completes the proof of Lemma 3.5.

We now use Lemma 3.5 to construct a smooth approximate steady-state solution for the case $K_{0} \sqrt{h} \leqq\left|M_{0}^{2}-1\right| \leqq h^{\beta} M_{0}^{2}, h \leqq h_{3}$, satisfying appropriate $L^{1}$ and $L^{\infty}$ properties, which will be used to construct the approximate solution sequence to (1.2) and to analyze its convergence and consistency.

Lemma 3.6. Consider the case $K_{0} \sqrt{h} \leqq\left|M_{0}^{2}-1\right| \leqq h^{\beta} M_{0}^{2}$ with $h \leqq h_{3}$. Then there exists an approximate steady-state solution $v(x)=(\rho(x), m(x))$ such that

$$
v(x)=v_{0}(1+\mathbf{O}(\sqrt{h})), \quad \frac{1}{h} \int_{x_{0}-\frac{h}{2}}^{x_{0}+\frac{h}{2}} v(x) d x=v_{0},
$$

and

$$
\begin{cases}w(v(x)) \leqq w\left(v_{0}\right)(1+C h), & M_{0}>0, \\ z(v(x)) \geqq z\left(v_{0}\right)(1+C h), & M_{0}<0,\end{cases}
$$


where bounds $\mathbf{O}(\sqrt{h})$ and $C$ depend only on bounds of $a(x)$ and $\rho_{0}+\left|\frac{m_{0}}{\rho_{0}}\right|$ and are independent of $M_{0}$.

Proof. We first determine $\tilde{x} \in\left(x_{0}-\frac{h}{2}, x_{0}+\frac{h}{2}\right)$ in the approximate steady-state solutions (3.18) such that (3.19) holds.

The identity (3.19) is trivial for $m(x)$ :

$$
\frac{1}{h} \int_{x_{0}-\frac{h}{2}}^{x_{0}+\frac{h}{2}} m(x) d x=m_{0} \frac{1}{h} \int_{x_{0}-\frac{h}{2}}^{x_{0}+\frac{h}{2}}\left(1-a_{0}\left(x-x_{0}\right)\right) d x=m_{0} .
$$

We now prove that there exists $\tilde{x} \in\left(x_{0}-\frac{h}{2}, x_{0}+\frac{h}{2}\right)$ such that

$$
\frac{1}{h} \int_{x_{0}-\frac{h}{2}}^{x_{0}+\frac{h}{2}} \rho(x) d x=\rho_{0}
$$

that is,

$$
\frac{1}{h} \int_{x_{0}-\frac{h}{2}}^{x_{0}+\frac{h}{2}}\left(1+\frac{M_{0}^{2}-1}{2(\theta+1)}\left(1-\sqrt{1+\frac{4(\theta+1) a_{0}}{\left(M_{0}^{2}-1\right)^{2}}(x-\tilde{x})}\right)\right) d x=1 .
$$

It suffices to prove that there exists $\tilde{x} \in\left(x_{0}-\frac{h}{2}, x_{0}+\frac{h}{2}\right)$ such that

$$
\frac{1}{h} \int_{x_{0}-\tilde{x}-\frac{h}{2}}^{x_{0}-\tilde{x}+\frac{h}{2}} \sqrt{1+\frac{4(\theta+1) a_{0}}{\left(M_{0}^{2}-1\right)^{2}}} x d x=1
$$

Set

$$
F(y)=\operatorname{sign}\left(a_{0}\right) \frac{1}{h} \int_{y-\frac{h}{2}}^{y+\frac{h}{2}}\left(\sqrt{1+\frac{4(\theta+1) a_{0}}{\left(M_{0}^{2}-1\right)^{2}} x}-1\right) d x
$$

Then

$$
F\left(\frac{h}{2}\right)>0, \quad F\left(-\frac{h}{2}\right)<0 .
$$

Furthermore, for $-\frac{h}{2} \leqq y \leqq \frac{h}{2}$, we have

$$
\begin{aligned}
F^{\prime}(y)=\frac{\operatorname{sign}\left(a_{0}\right)}{h}( & \sqrt{1+\frac{4(\theta+1) a_{0}}{\left(M_{0}^{2}-1\right)^{2}}\left(y+\frac{h}{2}\right)} \\
& \left.-\sqrt{1+\frac{4(\theta+1) a_{0}}{\left(M_{0}^{2}-1\right)^{2}}\left(y-\frac{h}{2}\right)}\right)>0 .
\end{aligned}
$$

We conclude that there exists $\tilde{x} \in\left(x_{0}-\frac{h}{2}, x_{0}+\frac{h}{2}\right)$ such that

$$
F\left(x_{0}-\tilde{x}\right)=0 \text {, }
$$

which proves (3.19). 
We now turn to (3.20). For the case $M_{0}>0$, we have

$$
\begin{aligned}
\frac{w(v(x))}{w\left(v_{0}\right)}= & \left(\frac{\rho}{\rho_{0}}\right)^{\theta} \frac{\theta M+1}{\theta M_{0}+1} \\
= & \left(\frac{\rho}{\rho_{0}}\right)^{-1}\left(1+\frac{\left(\frac{\rho}{\rho_{0}}\right)^{\theta+1}-1-\theta a_{0} M_{0}\left(x-x_{0}\right)}{\theta M_{0}+1}\right) \\
= & \left(1-\frac{M_{0}^{2}-1}{2(\theta+1)}\left(1-\sqrt{\left.1+\frac{4(\theta+1) a_{0}}{\left(M_{0}^{2}-1\right)^{2}(x-\tilde{x})}\right)}\right)\right. \\
& \times\left(1+\frac{M_{0}^{2}-1}{2(\theta+1)}\left(1-\sqrt{1+\frac{4(\theta+1) a_{0}}{\left(M_{0}^{2}-1\right)^{2}}(x-\tilde{x})}\right)\right) \\
& \left.+\mathbf{O}\left(\left|M_{0}^{2}-1\right|^{2} \mid 1-\sqrt{1+\frac{4(\theta+1) a_{0}}{\left(M_{0}^{2}-1\right)^{2}(x-\tilde{x})} \mid}\right)\left|x-x_{0}\right|\right) \\
= & 1+\mathbf{O}\left(\left|x-x_{0}\right|\right)+\mathbf{O}\left(\frac{|x-\tilde{x}|}{1+\sqrt{1+\frac{4(\theta+1) a_{0}}{\left(M_{0}^{2}-1\right)^{2}}(x-\tilde{x})}}\right) \leqq 1+C h,
\end{aligned}
$$

that is,

$$
w(v(x)) \leqq w\left(v_{0}\right)(1+C h),
$$

by using $w\left(v_{0}\right)>0$ in this case.

For the case $M_{0}<0$, we can similarly obtain

$$
\frac{z(v(x))}{z\left(v_{0}\right)} \leqq 1+C h
$$

which implies

$$
z(v(x)) \geqq z\left(v_{0}\right)(1+C h),
$$

by using $z\left(v_{0}\right)<0$.

3.3. The Transonic Shock. The case $\left|M_{0}^{2}-1\right|<K_{0} \sqrt{h}$ may require the introduction of a standing shock wave. This shock wave is part of the exact solution of the steady-state equations, and thus arrives in our construction while transferring boundary data, at the mesh block centers, to the mesh edges where a Riemann problem is solved. This situation is analyzed in Lemma 3.8. A similar occurrence of a standing shock arises in the introduction of source term corrections to the homogeneous Riemann solution. Here again, the steady-state solution is used to move constant states separating waves in the Riemann solution from its center at the mesh block edge to its final location at $t=(n+1) \Delta t$, propagated away from mesh block edges. This standing shock is located exactly at the mesh block edge, and is analyzed in Lemma 3.7. 
Lemma 3.7. Consider the case $\left|M_{0}^{2}-1\right|<K_{0} \sqrt{h}$ with $h \leqq h_{3}$. Then, for any $\tilde{x} \in\left[x_{0}-\frac{h}{2}, x_{0}+\frac{h}{2}\right]$, there exists an approximate steady-state solution, including a standing shock at $x=\tilde{x}$, in the sense of Definition 3.1 in the interval $\left[x_{0}-\frac{h}{2}, x_{0}+\frac{h}{2}\right]$, such that

$$
\begin{cases}w(v(x)) \leqq w\left(v_{0}\right)(1+C h), & M_{0}>0, \\ z(v(x)) \geqq z\left(v_{0}\right)(1+C h), & M_{0}<0,\end{cases}
$$

where the bounds $C$ and $\mathbf{o}(1)$ in Definition 3.1 depend only on bounds of $\left|\rho_{0}\right|+\left|\frac{m_{0}}{\rho_{0}}\right|$ and $a(x)$ and are independent of $M_{0}$.

Proof. We prove the lemma only for the case $M_{0}>0$. The other case can be proved in the same fashion.

Introduce a standing shock at $x=\tilde{x}$ with left state $v_{-}=\left(\rho_{0-}, m_{0}\right)$ and right state $v_{+}=\left(\rho_{0+}, m_{0}\right)$, where

$$
\rho_{0 \pm}=\rho_{0}\left(\frac{\theta M_{0}+1}{\theta+1}\right)^{\frac{1}{\theta}}\left(1 \pm K_{0} \sqrt{h}\right) .
$$

Then the corresponding Mach numbers are

$$
M_{0 \pm}^{2}=1 \mp 2(\theta+1) K_{0} \sqrt{h}+\mathbf{O}(h) .
$$

Therefore, we have

$$
\begin{aligned}
\frac{w\left(v_{ \pm}\right)}{w\left(v_{0}\right)} & =\left(\frac{\rho_{0 \pm}}{\rho_{0}}\right)^{\theta} \frac{\theta M_{0 \pm}+1}{\theta M_{0}+1} \\
& =\left(1 \pm \theta K_{0} \sqrt{h}\right)^{\theta} \frac{1+\theta \sqrt{1 \mp 2(\theta+1) K_{0} \sqrt{h}+\mathbf{O}(h)}}{\theta+1} \\
& =1-\theta^{2} K_{0}^{2} h+\mathbf{O}(h) \leqq 1+C h .
\end{aligned}
$$

Define

$$
\rho_{ \pm}(x)=\rho_{0 \pm}\left(1+\frac{M_{0 \pm}^{2}-1}{2(\theta+1)}\left(1-\sqrt{1+\frac{4(\theta+1) a_{0}}{\left(M_{0 \pm}^{2}-1\right)^{2}}(x-\tilde{x})}\right)\right) .
$$

Then $v(x)=(\rho(x), m(x))$ determined by

$$
\left\{\begin{array}{l}
\rho(x)=\left\{\begin{array}{l}
\rho_{-}(x), \quad x \in\left[x_{0}-\frac{h}{2}, \tilde{x}\right), \\
\text { standing shock, } \quad x=\tilde{x}, \\
\rho_{+}(x), \quad x \in\left[\tilde{x}, x_{0}+\frac{h}{2}\right],
\end{array}\right. \\
m(x)=m_{0}\left(1-a_{0}\left(x-x_{0}\right)\right),
\end{array}\right.
$$

is an approximate solution satisfying

$$
f(v)_{x}+a(x) g(v)=\mathbf{O}\left(h^{\beta}\right)
$$

in the sense of Definition 3.1.

Furthermore, using Lemma 3.6, we have

$$
\frac{w\left(v_{ \pm}(x)\right)}{w\left(v_{0 \pm}\right)} \leqq 1+C h .
$$


Therefore, we obtain

$$
\frac{w(v(x))}{w\left(v_{0}\right)}= \begin{cases}\frac{w\left(v_{+}(x)\right)}{w\left(v_{0+}\right)} \frac{w\left(v_{0+}\right)}{w\left(v_{0}\right)} \leqq 1+C h, & x \in\left[x_{0}-\frac{h}{2}, \tilde{x}\right), \\ \frac{w\left(v_{-}(x)\right)}{w\left(v_{0-}\right)} \frac{w\left(v_{0-}\right)}{w\left(v_{0}\right)} \leqq 1+C h, & x \in\left(\tilde{x}, x_{0}+\frac{h}{2}\right]\end{cases}
$$

This completes the proof of Lemma 3.7.

Now we introduce the second approximate steady-state solution for the case $\left|M_{0}^{2}-1\right|<K_{0} \sqrt{h}$. It plays an important role in constructing the approximate solutions and ensures the consistency of the approximate solution with the system (1.2).

Lemma 3.8. There exist $h_{4}>0$ and an approximate steady-state solution, including a standing shock at some $\tilde{x} \in\left(x_{0}-\frac{2+\theta}{4(1+\theta)} h, x_{0}+\frac{2+\theta}{4(1+\theta)} h\right)$ with $h \leqq h_{4}$, in the sense of Definition 3.1, such that, when $\left|M_{0}^{2}-1\right|<K_{0} \sqrt{h}$,

$$
v(x)=v_{0}(1+\mathbf{O}(\sqrt{h})), \quad \frac{1}{h} \int_{x_{0}-\frac{h}{2}}^{x_{0}+\frac{h}{2}} v(x) d x=v_{0},
$$

where the bound $\mathbf{O}(1)$ depends only on $\left|\rho_{0}\right|+\left|\frac{m_{0}}{\rho_{0}}\right|$ and $a(x)$ and are independent of $M_{0}$.

Proof. Using Lemma 3.7, we construct an approximate steady-state solution $v(x ; \tilde{x})$ in the sense of Definition 3.1, including a standing shock at some point $\tilde{x} \in\left(x_{0}-\right.$ $\left.\frac{2+\theta}{4(1+\theta)} h, x_{0}+\frac{2+\theta}{4(1+\theta)} h\right)$, such that $m(x, \tilde{x})=m(x)$ determined by (3.18), namely,

$$
\left.m(x ; \tilde{x})\right|_{x=\tilde{x}}=m_{0}\left(1-a_{0}\left(\tilde{x}-x_{0}\right)\right) .
$$

The identity (3.21) for $m(x, \tilde{x})$ is clear as in Lemma 3.6. Therefore, the main point is to choose $\tilde{x} \in\left(x_{0}-\frac{2+\theta}{4(1+\theta)} h, x_{0}+\frac{2+\theta}{4(1+\theta)} h\right)$ such that $(3.21)$ also holds for $\rho(x ; \tilde{x})$. We prove only for the case $M_{0}>0$ and thus $M_{0 \pm}>0$. The other case can be proved in the same way.

Notice that

$$
\frac{1}{h} \int_{x_{0}-\frac{h}{2}}^{x_{0}+\frac{h}{2}} \rho(x ; \tilde{x}) d x-\rho_{0}=\rho_{0} G\left(x_{0}-\tilde{x}\right)
$$

where

$$
\begin{aligned}
G(y)= & \left(\frac{\theta M_{0}+1}{\theta+1}\right)^{\frac{1}{\theta}} \\
& \times\left\{\begin{array}{l}
\frac{1-K_{0} \sqrt{h}}{h} \int_{y-\frac{h}{2}}^{0}\left(1+\frac{M_{0-}^{2}-1}{2(\theta+1)}\left(1-\sqrt{1+\frac{4(\theta+1) a_{0}}{\left(M_{0-}^{2}-1\right)^{2}} x}\right)\right) d x \\
+\frac{1+K_{0} \sqrt{h}}{h} \int_{0}^{y+\frac{h}{2}}\left(1+\frac{M_{0+}^{2}-1}{2(\theta+1)}\left(1-\sqrt{1+\frac{4(\theta+1) a_{0}}{\left(M_{0+}^{2}-1\right)^{2}} x}\right)\right) d x
\end{array}\right\}-1,
\end{aligned}
$$

for $y \in\left[-\frac{2+\theta}{4(1+\theta)} h, \frac{2+\theta}{4(1+\theta)} h\right]$. 
A direct calculation shows that there exists $h_{4}>0$ such that, when $h \leqq h_{4}$, we have

$$
\left\{\begin{array}{l}
G^{\prime}(y)>0, \quad y \in\left(-\frac{2+\theta}{4(1+\theta)} h, \frac{2+\theta}{4(1+\theta)} h\right), \\
G\left(\frac{2+\theta}{4(1+\theta)} h\right) G\left(-\frac{2+\theta}{4(1+\theta)} h\right)<0
\end{array}\right.
$$

using the choice of $K_{0}$ determined by (3.17), where $h_{4}>0$ depends only on $\|a\|_{C}$.

In fact, using

$$
M_{0 \pm}^{2}-1=\mp 2(\theta+1) K_{0} \sqrt{h}+\mathbf{O}(h) \text {, }
$$

we have

$$
\begin{aligned}
G(y)= & \left(\frac{\theta M_{0}+1}{\theta+1}\right)^{\frac{1}{\theta}} \\
& \times\left\{\begin{array}{c}
\left(1-K_{0} \sqrt{h}\right) \int_{\frac{y}{h}-\frac{1}{2}}^{0}\left(1+K_{0} \sqrt{h}(1-\sqrt{1+B x})\right) d x \\
+\left(1+K_{0} \sqrt{h}\right) \int_{0}^{\frac{y}{h}+\frac{1}{2}}\left(1-K_{0} \sqrt{h}(1-\sqrt{1+B x})\right) d x
\end{array}\right\}-1+\mathbf{O}(h) \\
= & \frac{K_{0} \sqrt{h}}{\theta+1}\left\{\frac{M_{0}-1}{K_{0} \sqrt{h}}+(\theta+1)\left(\int_{0}^{\frac{y}{h}+\frac{1}{2}} \sqrt{1+B x} d x-\int_{\frac{y}{h}-\frac{1}{2}}^{0} \sqrt{1+B x} d x\right)\right\}+\mathbf{O}(h) \\
= & \frac{K_{0} \sqrt{h}}{\theta+1}\left\{\frac{M_{0}-1}{K_{0} \sqrt{h}}+\Delta(y)\right\}+\mathbf{O}(h),
\end{aligned}
$$

where

and

$$
B=\frac{a_{0}}{(\theta+1) K_{0}^{2}}
$$

$$
\Delta(y)=(\theta+1)\left\{\int_{0}^{\frac{y}{h}+\frac{1}{2}} \sqrt{1+B x} d x-\int_{\frac{y}{h}-\frac{1}{2}}^{0} \sqrt{1+B x} d x\right\} .
$$

Using

$$
(1+x)^{\frac{3}{2}}=1+\frac{3}{2} x+\frac{3}{4} x^{2} \int_{0}^{1} \frac{1-\tau}{\sqrt{1+\tau x}} d \tau, \quad|x|<1,
$$

we have, for $0<\alpha<1$,

$$
\begin{aligned}
\Delta\left( \pm \alpha \frac{h}{2}\right)=\frac{2(\theta+1)}{3 B} & \left\{\left(1+B\left(\frac{1 \pm \alpha}{2}\right)\right)^{\frac{3}{2}}+\left(1-B\left(\frac{1 \mp \alpha}{2}\right)\right)^{\frac{3}{2}}-2\right\} \\
=(\theta+1)\{ & \pm \alpha+\frac{B}{8}\left((1 \pm \alpha)^{2} \int_{0}^{1} \frac{1-\tau}{\sqrt{1+\frac{\tau}{2}(1 \pm \alpha) B}} d \tau\right. \\
& \left.\left.+(1 \mp \alpha)^{2} \int_{0}^{1} \frac{1-\tau}{\sqrt{1-\frac{\tau}{2}(1 \mp \alpha) B}} d \tau\right)\right\} .
\end{aligned}
$$

Noting

$$
1-B \geqq \frac{1}{4},
$$


from (3.17), we have

$$
\Delta\left(\alpha \frac{h}{2}\right)>(\theta+1) \alpha-\frac{\alpha^{2}+1}{K_{0}^{2}}\left|a_{0}\right| \geqq 1+(\theta+1)\left(\alpha-\frac{2+\theta}{2(1+\theta)}\right)=1,
$$

and

$$
\Delta\left(-\alpha \frac{h}{2}\right)<-(\theta+1) \alpha+\frac{\alpha^{2}+1}{K_{0}^{2}}\left|a_{0}\right| \leqq-1-(\theta+1)\left(\alpha-\frac{2+\theta}{2(1+\theta)}\right)=-1
$$

by choosing $\alpha=\frac{2+\theta}{2(1+\theta)}<1$. This means that

$$
G\left(\frac{2+\theta}{4(1+\theta)} h\right) G\left(-\frac{2+\theta}{4(1+\theta)} h\right)<0,
$$

by using $\left|\frac{M_{0}-1}{K_{0} \sqrt{h}}\right| \leqq 1$ for this case.

On the other hand, one can easily check that

$$
G^{\prime}(y)=\frac{K_{0}}{\sqrt{h}}\left(\sqrt{1+B\left(\frac{y}{h}+\frac{1}{2}\right)}+\sqrt{1+B\left(\frac{y}{h}-\frac{1}{2}\right)}+\mathbf{O}(h)\right) .
$$

Therefore, there exists $h_{4}>0$ such that, when $h<h_{4}$, one has

$$
G^{\prime}(y)>0, \quad y \in\left(x_{0}-\frac{2+\theta}{4(1+\theta)} h, x_{0}+\frac{2+\theta}{4(1+\theta)} h\right) .
$$

We conclude that there exists $\tilde{x} \in\left(x_{0}-\frac{2+\theta}{4(1+\theta)} h, x_{0}+\frac{2+\theta}{4(1+\theta)} h\right)$ such that

$$
G\left(x_{0}-\tilde{x}\right)=0
$$

that is,

$$
\frac{1}{h} \int_{x_{0}-\frac{h}{2}}^{x_{0}+\frac{h}{2}} \rho(x ; \tilde{x}) d x=\rho_{0} .
$$

This completes the proof of Lemma 3.8.

\section{Approximate Solutions}

In this section we construct approximate solutions $v^{h}=\left(\rho^{h}, m^{h}\right)=\left(\rho^{h}, \rho^{h} u^{h}\right)$ in the strip $0 \leqq t \leqq T$ for any fixed $T \in(0, \infty)$, where

$$
h \leqq h_{0} \equiv \min _{1 \leqq i \leqq 4} h_{i}
$$

is the space mesh length, together with the time mesh length $\Delta t$, satisfying the following Courant-Friedrichs-Levy condition:

$$
\Lambda \equiv \max _{i=1,2}\left(\sup _{0 \leqq t \leqq T}\left|\lambda_{i}\left(\rho^{h}, m^{h}\right)\right|\right) \leqq \frac{\gamma-1}{4(\gamma+1)} \frac{h}{\Delta t} \leqq 2 \Lambda,
$$


where the factor $\frac{\gamma-1}{4(\gamma+1)}$ prevents interaction among the waves in the Riemann solutions starting at the mesh cell edges with the standing shocks resulting from solutions of the steady-state equations to transfer data from cell centers. We will prove that the approximate solutions are bounded uniformly in the mesh length $h>0$ and $\rho^{h}(x, t) \geqq 0$ to guarantee the construction of $\left(\rho^{h}, m^{h}\right)$. Since the speeds of propagation of the approximate solutions will be finite because of (4.2), we can assume that the initial data have compact support (i.e. are constant off some compact set) without loss of generality.

\subsection{Construction of Approximate Solutions}

A. Initial Value Problem. We first construct the approximate solutions $\left(\rho^{h}, m^{h}\right)$ for the Cauchy problem. Let

$$
t_{n}=n \Delta t, \quad x_{j}=j h, \quad(n, j) \in \mathbf{Z}_{+} \times \mathbf{Z} .
$$

Assume that $v^{h}(x, t)$ is defined for $t<n \Delta t$. Then we define $v_{j}^{n} \equiv\left(\rho_{j}^{n}, m_{j}^{n}\right)$ as

$$
\left\{\begin{array}{l}
\rho_{j}^{n} \equiv \max \left(\frac{1}{h} \int_{\left(j-\frac{1}{2}\right) h}^{\left(j+\frac{1}{2}\right) h} \rho^{h}(x, n \Delta t-0) d x, h^{\beta}\right), \quad\left(j-\frac{1}{2}\right) h \leqq x \leqq\left(j+\frac{1}{2}\right) h, \\
m_{j}^{n} \equiv \frac{1}{h} \int_{\left(j-\frac{1}{2}\right) h}^{\left(j+\frac{1}{2}\right) h} m^{h}(x, n \Delta t-0) d x, \quad\left(j-\frac{1}{2}\right) h \leqq x \leqq\left(j+\frac{1}{2}\right) h,
\end{array}\right.
$$

where $\beta \in\left(0, \frac{1}{6}\right)$ is the same constant as in Lemma 3.2.

In the strip $n \Delta t \leqq t<(n+1) \Delta t, v^{h}(x, t)$ is defined as an approximate solution of the Cauchy problem

$$
\left\{\begin{array}{l}
v_{t}+f(v)_{x}=a(x) g(v), \quad j h \leqq x \leqq(j+1) h, \\
\left.v\right|_{t=n \Delta t}= \begin{cases}v_{-}(x), & x<\left(j+\frac{1}{2}\right) h \\
v_{+}(x), & x>\left(j+\frac{1}{2}\right) h\end{cases}
\end{array}\right.
$$

where $v_{-}(x)$ and $v_{+}(x)$ are smooth solutions of the steady-state equations (3.1) with boundary conditions

$$
v_{-}(j h)=v_{j}^{n}, \quad v_{+}((j+1) h)=v_{j+1}^{n},
$$

constructed in Sect. 3.1 when $\left|M^{2}\left(v_{k}^{n}\right)-1\right| \geqq h^{\beta} M^{2}\left(v_{k}^{n}\right), k=j, j+1$, and, otherwise, are approximate steady-state solutions constructed in Lemma 3.6 and Lemma 3.8 in Sect. 3.2. Then the difference between the average of the exact or approximate steady-state solutions over $\left[\left(j-\frac{1}{2}\right) h,\left(j+\frac{1}{2}\right) h\right]$ and the Godunov value $v_{j}^{n}$ is higher than first order in $h$ by Lemma 3.4, as the Godunov value away from the sonic state and, otherwise, by Lemmas 3.6 and 3.8. Such a construction ensures the consistency of the corresponding approximate solutions with the Euler equations.

We will solve the problem for small time approximately. This is done by perturbing about the solution of the Riemann problem at $x=\left(j+\frac{1}{2}\right) h$ :

$$
\left\{\begin{array}{l}
v_{t}+f(v)_{x}=0, \quad j h \leqq x<(j+1) h, \\
\left.v\right|_{t=n \Delta t}= \begin{cases}v_{-}\left(\left(j+\frac{1}{2}\right) h-0\right), & x<\left(j+\frac{1}{2}\right) h \\
v_{+}\left(\left(j+\frac{1}{2}\right) h+0\right), & x>\left(j+\frac{1}{2}\right) h .\end{cases}
\end{array}\right.
$$


We first construct $R_{a}(x, t)$ as an approximate Riemann solution to (4.5), which excludes the vacuum state. We obtain $R_{a}(x, t)$ by finding all possible interaction points with the line $\rho=h^{\beta}$ in the phase plane $(\rho, m)$. We introduce, if necessary, an extra constant region with $\rho=h^{\beta}$ in solving the Riemann problem. Thus $R_{a}(x, t)$ satisfies the entropy condition on its discontinuities and has the relation

$$
\left|R_{a}(x, t)-R(x, t)\right| \begin{cases}=0, & \text { when } \rho(x, t) \geqq h^{\beta}, \\ \leqq C h^{\beta}, & \text { otherwise },\end{cases}
$$

with the exact Riemann solution $R(x, t)$. Following the ideas of [D1], we approximate possible existing $\kappa^{\text {th }}$ rarefaction waves $\left(v_{-}^{r}, v_{+}^{r}\right), \kappa=1,2$, in $R_{a}(x, t)$ by finite discontinuous rays. The advantage of this method for our problem is that it facilitates estimates of source term corrections to Riemann solutions. We introduce the rays $\frac{x_{i}}{t}=\lambda_{\kappa}\left(v_{i}^{r}\right)$ separating finite constant states $v_{i}^{r}, i=0,1, \ldots, m_{r}$, with $v_{0}^{r}=v_{-}^{r}$ and $v_{m_{r}}^{r}=v_{+}^{r}$, such that

$$
w\left(v_{i+1}^{r}\right)=w\left(v_{i}^{r}\right)+h, \quad z\left(v_{i+1}^{r}\right)=z\left(v_{i}^{r}\right), \quad 0 \leqq i \leqq m_{r}-1,
$$

if $\left(v_{-}^{r}, v_{+}^{r}\right)$ is the 1 -rarefaction wave with $\kappa=1$; or

$$
z\left(v_{i+1}^{r}\right)=z\left(v_{i}^{r}\right)+h, \quad w\left(v_{i+1}^{r}\right)=w\left(v_{i}^{r}\right), \quad 0 \leqq i \leqq m_{r}-1,
$$

if $\left(v_{-}^{r}, v_{+}^{r}\right)$ is the 2-rarefaction wave with $\kappa=2$. In this way, we obtain the approximate Riemann solutions consisting of finite discontinuities separating finite constant states $v_{i}, i=0,1, \ldots, I$, with $v_{0}=v_{-}\left(\left(j+\frac{1}{2}\right) h-0\right)$ and $v_{I}=v_{+}\left(\left(j+\frac{1}{2}\right) h+0\right)$. To control local entropy errors on the discontinuities in the approximate solutions (as in Lemmas 4.1-4.2 we establish below), we need a lower bound for the density in the approximate solutions. For this purpose, let $\bar{v}_{i}(x)=\left(\bar{\rho}_{i}(x), \bar{m}_{i}(x)\right), 1 \leqq i \leqq I-1$, with $\bar{v}_{0}=v_{-}(x)$ and $\bar{v}_{I}=v_{+}(x)$, be the exact steady-state solutions satisfying the boundary conditions

$$
\left.\bar{v}_{i}(x)\right|_{x=\left(j+\frac{1}{2}\right) h}=v_{i}, \quad 0 \leqq i \leqq I-1,
$$

determined by the smooth steady-state solutions in Sect. 3.1 if $\left|M^{2}\left(v_{i}\right)-1\right| \geqq$ $h^{\beta} M^{2}\left(v_{i}\right)$ and are, otherwise, approximate steady-state solutions constructed by Lemmas 3.6-3.7 in Sect. 3.2. We use the cut-off technique and denote by $v_{i}(x)=$ $\left(\rho_{i}(x), \rho_{i}(x) u_{i}(x)\right), 0 \leqq i \leqq I$, the approximate steady-state solutions as follows:

$$
\rho_{i}(x)=\max \left(\bar{\rho}_{i}(x), h^{\beta}\right), \quad u_{i}(x)=\bar{u}_{i}(x) \equiv \frac{\bar{m}_{i}(x)}{\bar{\rho}_{i}(x)}, \quad 0 \leqq i \leqq I .
$$

The approximate solution $\left(\rho^{h}(x, t), m^{h}(x, t)\right)$ of the Cauchy problem (4.4) in the rectangle $[j h,(j+1) h] \times[n \Delta t,(n+1) \Delta t)$ consists of the (exact or approximate) steady states $v_{i}(x), i=0,1, \ldots, I$, separated by the discontinuities, subject to the Rankine-Hugoniot condition, with speeds

$$
\frac{d x(t)}{d t}=v_{i}(x(t), t)+(-1)^{\kappa} \sqrt{\frac{\rho_{i+1}(x(t), t)}{\rho_{i}(x(t), t)} \frac{p\left(\rho_{i+1}(x(t), t)\right)-p\left(\rho_{i}(x(t), t)\right)}{\rho_{i+1}(x(t), t)-\rho_{i}(x(t), t)}},
$$

where $\kappa=1$ or $\kappa=2$, determined by the $\kappa^{\text {th }}$ original elementary waves from which the discontinuity comes. Then the approximate solutions $\left(\rho^{h}(x, t), m^{h}(x, t)\right)$ approach the approximate Riemann solutions as $t \rightarrow n \Delta t$. 
B. Initial-Boundary Problem. Assume that $v^{h}(x, t)$ is defined for $t<n \Delta t$. Now we construct $v^{h}(x, t)$ in the strip $[n \Delta t,(n+1) \Delta t)$. For $j>1$, the construction is as in Sect. 4.1.A in the rectangle $\left[\left(j-\frac{1}{2}\right) h+1,\left(j+\frac{1}{2}\right) h+1\right] \times[n \Delta t,(n+1) \Delta t]$. Now we consider the boundary interval $\left[1,1+\frac{3}{2} h\right]$ associated with $j=1$. For $j=1$, let

$$
v^{h}(x, n \Delta t+0)=v_{1}^{n} \equiv\left\{\begin{array}{l}
\rho_{1}^{n} \equiv \max \left(\frac{2}{3 h} \int_{1}^{1+\frac{3}{2} h} \rho^{h}(x, n \Delta t-0) d x, h^{\beta}\right) \\
m_{1}^{n} \equiv \frac{2}{3 h} \int_{1}^{1+\frac{3}{2} h} m^{h}(x, n \Delta t-0) d x
\end{array}\right.
$$

for $1 \leqq x \leqq 1+\frac{3}{2} h$. Denote $v_{1}(x)$ as the (exact or approximate) solution of the steady-state equations (3.1) with initial conditions

$$
v_{1}(1+h)=v_{1}^{n}
$$

constructed in Sect. 3. In the strip $n \Delta t \leqq t<(n+1) \Delta t, v^{h}(x, t)$ is defined as the approximate solution of the corresponding generalized Riemann initial-boundary problem with initial value $v_{1}(x)$ at $t=n \Delta t$ over $[1,1+h]$. This is achieved by solving the problem for small time approximately as in Sect. 4.1.A (e.g. [MT]).

The approximate solution $\left(\rho^{h}(x, t), m^{h}(x, t)\right)$ of the Cauchy problem (4.3) in the rectangle $[1+j h, 1+(j+1) h] \times[n \Delta t,(n+1) \Delta t)$ or $\left[1,1+\frac{3}{2} h\right] \times[n \Delta t,(n+1) \Delta t)$ consists of the steady states $v_{i}(x), i=0,1, \ldots, m_{r}$, separated by the discontinuities with speeds

$$
\sigma_{i+1}=\frac{d x(t)}{d t} \equiv v_{i}(x(t), t)+(-1)^{\kappa} \sqrt{\frac{\rho_{i+1}(x(t), t)}{\rho_{i}(x(t), t)} \frac{p\left(\rho_{i+1}(x(t), t)\right)-p\left(\rho_{i}(x(t), t)\right)}{\rho_{i+1}(x(t), t)-\rho_{i}(x(t), t)}}
$$

where $\kappa=1$, or $\kappa=2$, determined by the $\kappa^{\text {th }}$ original elementary waves from which the discontinuity comes. Then the approximate solution $\left(\rho^{h}(x, t), m^{h}(x, t)\right)$ approaches the approximate Riemann solution as $t \rightarrow n \Delta t$.

4.2. Local Entropy Estimates. We now estimate local entropy errors of the discontinuities in the approximate solutions to allow the proof of vanishing of global entropy errors in the context of the $H^{-1}$ compactness estimates in Sect. 6 and the consistency proof in Sect. 7 of the weak limit solution.

Lemma 4.1. On the discontinuous rays, $x=x_{i}(t), \sigma_{i}=\frac{d x_{i}(t)}{d t}$, of the approximate rarefaction waves constructed in Sect. 4.1,

$$
\left|\sigma_{i}\left[\eta\left(v_{i+1}(x(t), t)\right)-\eta\left(v_{i}(x(t), t)\right)\right]-\left[q\left(v_{i+1}(x(t), t)\right)-q\left(v_{i}(x(t), t)\right)\right]\right| \leqq C h^{3-2 \beta},
$$

for any $C^{2}$ weak entropy-entropy flux pair $(\eta, q)$, where $C$ is a constant depending only on the uniform bound of $v^{h}(x, t)$.

Proof. It suffices to prove that (4.12) holds at any point of the corresponding discontinuity wave curve in the neighborhood of $v_{i}(x(t))$ for each fixed $t$.

Denote $v_{0}=\left(\rho_{0}, m_{0}\right) \equiv v_{i}\left(x\left(t_{0}\right)\right)$ as a left state of the discontinuity wave curve and denote $v=(\rho, m)$ as a point on the corresponding discontinuity wave curve 
with the left state $v_{0}$ at some time $t_{0}$. Along the wave curve, we have from (2.4),

$$
\begin{aligned}
& m(\rho)=\frac{m_{0}}{\rho_{0}} \rho \pm \sqrt{\frac{\rho}{\rho_{0}} \frac{p(\rho)-p\left(\rho_{0}\right)}{\rho-\rho_{0}}}\left(\rho-\rho_{0}\right), \\
& \sigma(\rho)=\frac{m(\rho)-m_{0}}{\rho-\rho_{0}}=\frac{m_{0}}{\rho_{0}} \pm \sqrt{\frac{\rho}{\rho_{0}} \frac{p(\rho)-p\left(\rho_{0}\right)}{\rho-\rho_{0}}} .
\end{aligned}
$$

Set

$$
Q(\rho)=\sigma(\rho)\left(\eta(v(\rho))-\eta\left(v_{0}\right)\right)-\left(q(v(\rho))-q\left(v_{0}\right)\right)
$$

Then

$$
\dot{Q}(\rho)=\dot{\sigma}(\rho)\left(\eta(v(\rho))-\eta\left(v_{0}\right)\right)+\sigma(\rho) \dot{\eta}(v(\rho))-\dot{q}(v(\rho))
$$

Notice that

$$
\left\{\begin{array}{l}
\dot{\sigma}(\rho)\left(v(\rho)-v_{0}\right)+\sigma(\rho) \dot{v}(\rho)=\dot{f}(v(\rho)), \quad \text { (Rankine-Hugoniot condition) } \\
\dot{q}(v(\rho))=\nabla q \cdot \dot{v}(\rho)=\nabla \eta \dot{f}(v(\rho))
\end{array}\right.
$$

We have

$$
\begin{aligned}
\dot{Q}(\rho) & =\dot{\sigma}(\rho)\left(\eta(v(\rho))-\eta\left(v_{0}\right)-\nabla \eta(v(\rho))\left(v(\rho)-v_{0}\right)\right) \\
& =-\dot{\sigma}(\rho) \int_{0}^{1} \tau \frac{d^{2}}{d \tau^{2}} \eta\left(v_{0}+\tau\left(v(\rho)-v_{0}\right)\right) d \tau
\end{aligned}
$$

Therefore, using (4.7)-(4.8), Lemma 3.4, Lemma 3.6, Lemma 3.8, and the property of the Rankine-Hugoniot locus, we obtain

$$
\begin{aligned}
|Q(\rho)| & =\left|\int_{\rho_{0}}^{\rho} \dot{Q}(s) d s\right| \\
& =\left|\int_{\rho_{0}}^{\rho}(-\dot{\sigma}(s))\left(\eta(v(s))-\eta\left(v_{0}\right)-\nabla \eta(v(s))\left(v(s)-v_{0}\right)\right) d s\right| \\
& =\left|\int_{\rho_{0}}^{\rho} \dot{\sigma}(s) d s \int_{0}^{1} \tau\left(v(s)-v_{0}\right)^{\top} \nabla^{2} \eta\left(v_{0}+\tau\left(v(s)-v_{0}\right)\right)\left(v(s)-v_{0}\right) d \tau\right| \\
& \leqq C \int_{\rho_{0}}^{\rho}|\dot{\sigma}(s)| d s \int_{0}^{1} \tau\left(v(s)-v_{0}\right)^{\top} \nabla^{2} \eta_{*}\left(v_{0}+\tau\left(v(s)-v_{0}\right)\right)\left(v(s)-v_{0}\right) d \tau \\
& \leqq C\left|\rho-\rho_{0}\right| \sup _{\rho \in\left[\rho_{0}, \rho\right]}\left|v(\rho)-v_{0}\right|^{2}\left(\min \left(\rho, \rho_{0}\right)\right)^{-2} \leqq C h^{\frac{3}{2}-2 \beta}
\end{aligned}
$$

Lemma 4.2. There is a constant $C$ depending only on the uniform bound of $v^{h}(x, t)$ such that, on the approximate shock waves,

$$
\sigma_{i}(t)\left(\eta_{*}\left(v_{i+1}\right)-\eta_{*}\left(v_{i}\right)\right)-\left(q_{*}\left(v_{i+1}\right)-q_{*}\left(v_{i}\right)\right) \geqq 0,
$$


and

$$
\begin{aligned}
& \mid\left\{\sigma_{i}(t)\left(\eta\left(v_{i+1}(x(t), t)\right)-\eta\left(v_{i}(x(t), t)\right)\right)-\left(q\left(v_{i+1}(x(t), t)\right)-q\left(v_{i}(x(t), t)\right)\right)\right\} \\
& -\left\{\sigma_{i}(t)\left(\eta_{*}\left(v_{i+1}\right)-\eta_{*}\left(v_{i}\right)\right)-\left(q_{*}\left(v_{i+1}\right)-q_{*}\left(v_{i}\right)\right)\right\} \mid \leqq C h^{\frac{3}{2}-2 \beta},
\end{aligned}
$$

for any $C^{2}$ weak entropy-entropy flux pair $(\eta, q)$ satisfying (1.7) and the mechanical energy-energy flux $\left(\eta_{*}, q_{*}\right)$ defined by (1.8).

The proof of (4.14) and (4.15) is the same as the arguments leading to (4.13).

\section{5. $L^{\infty}$ Estimates}

We first derive an $L^{\infty}$ bound for the approximate solutions $v^{h}(x, t)$ of the Cauchy problem (1.3) and (1.5) and initial-boundary value problem (1.3) and (1.6), with the aid of the analysis of the approximate solutions $v^{h}(x, t)=\left(\rho^{h}(x, t), m^{h}(x, t)\right)$ constructed in Sect. 4. For any $T \in(0, \infty)$, define $\Pi_{T}=\mathbf{R} \times[0, T]$ for the Cauchy problem and $\Pi_{T}=[1, \infty) \times[0, T]$ for the initial-boundary value problem.

Theorem 5.1. Assume that $A(x)$ is a $C^{2}$ function bounded away from zero for all $x$ and that the initial velocity and nonnegative density data $\left(u_{0}, \rho_{0}\right)$ are bounded in $L^{\infty}$. Then, when $h \leqq h_{0}$ with $h_{0}$ determined by (4.1), the difference approximate solutions $\left(\rho^{h}, m^{h}\right)$ of the Cauchy problem (1.3) and (1.5) or initial-boundary value problem (1.3) and (1.6) with (1.4) are uniformly bounded in the region $\Pi_{T}$. That is, there exists a constant $C(T)>0$ such that

$$
\left|u^{h}(x, t)\right| \leqq C(T), \quad h^{\beta} \leqq \rho^{h}(x, t) \leqq C(T), \quad(x, t) \in \Pi_{T} .
$$

Proof. We prove only for the Cauchy problem (1.3) and (1.5). The proof for the initial-boundary problem (1.3) and (1.6) is the same.

First, using Lemma 2.1 and the construction of $\left(\rho^{h}, m^{h}\right)$, one immediately concludes that

$$
\rho^{h}(x, t) \geqq h^{\beta}, \quad \text { for }-\infty<x<\infty, \quad 0 \leqq t \leqq T .
$$

Now we make other estimates. For $n \Delta t \leqq t<(n+1) \Delta t, n \geqq 0$ integers, we use Lemma 2.1 and Lemmas 3.2-3.7, and the construction of $\left(\rho^{h}, m^{h}\right)$ to get

$$
\left\{\begin{array}{l}
w\left(v^{h}(x, t)\right) \leqq \max \left(\sup _{x} w\left(v^{h}(x, n \Delta t+0)\right), 1\right)(1+C \Delta t), \\
z\left(v^{h}(x, t)\right) \geqq \min \left(\inf _{x} z\left(v^{h}(x, n \Delta t+0)\right),-1\right)(1+C \Delta t),
\end{array}\right.
$$

for $h \leqq h_{0}$. In particular, this implies

$$
\left\{\begin{array}{l}
w\left(v^{h}(x,(n+1) \Delta t-0)\right) \leqq \max \left(\sup _{x} w\left(v^{h}(x, n \Delta t+0)\right), 1\right)(1+C \Delta t), \\
z\left(v^{h}(x,(n+1) \Delta t-0)\right) \geqq \min \left(\inf _{x} z\left(v^{h}(x, n \Delta t+0)\right),-1\right)(1+C \Delta t) .
\end{array}\right.
$$

Set $M_{n}=\max \left(\sup _{x} w\left(v^{h}(x, n \Delta t+0)\right),-\inf _{x} z\left(v^{h}(x, n \Delta t+0)\right), 1\right)$. Then one has $\max \left(\sup _{x} w\left(v^{h}(x,(n+1) \Delta t-0)\right),-\inf _{x} z\left(v^{h}(x,(n+1) \Delta t-0)\right)\right) \leqq M_{n}(1+C \Delta t)$. 
It follows from Lemma 2.1, Lemmas 3.2-3.4, and Lemmas 3.5-3.7 that

$$
M_{n+1} \leqq M_{n}(1+C \Delta t)
$$

that is,

$$
\frac{M_{n+1}-M_{n}}{\Delta t} \leqq C M_{n}
$$

Consider the corresponding ordinary differential equation

$$
\left\{\begin{array}{l}
\frac{d r}{d t}=C r \\
r(0)=r_{0} \equiv \max \left(\sup _{x} w\left(v_{0}(x)\right),-\inf _{x} z\left(v_{0}(x)\right), 1\right) .
\end{array}\right.
$$

It follows that

$$
r_{0} \leqq r(t) \leqq \tilde{C}(T) \equiv r_{0} e^{C T}, \quad \text { for } 0 \leqq t \leqq T .
$$

Noting that the integral curve $r=r(t)$ is a convex curve, we obtain from (5.2)(5.5) that

$$
M_{n} \leqq r(n \Delta t) \leqq \tilde{C}(T) .
$$

We derive from (5.2) and (5.6) that

$$
\left\{\begin{array}{l}
w\left(v^{h}(x, t)\right) \leqq \tilde{C}, \quad-z\left(v^{h}(x, t)\right) \leqq \tilde{C}, \\
w\left(v^{h}(x, t)\right)-z\left(v^{h}(x, t)\right) \geqq \frac{2 h^{\beta \theta}}{\theta},
\end{array}\right.
$$

that is, for $h \leqq h_{0}$, there is a constant $C(T)>0$ such that

$$
\left|u^{h}(x, t)\right|=\left|\frac{m^{h}(x, t)}{\rho^{h}(x, t)}\right| \leqq C, \quad h^{\beta} \leqq \rho^{h}(x, t) \leqq C .
$$

\section{6. $H^{-1}$ Compactness Estimates}

We now prove the $H^{-1}$ compactness for the approximate solutions $\left(\rho^{h}, m^{h}\right)$ of the Cauchy problem (1.3) and (1.5). We first introduce a basic lemma of functional analysis (see $[\mathrm{Mu}, \mathrm{Ta}, \mathrm{C} 1])$.

Lemma 6.1. Let $\Omega \subset R^{n}$ be a bounded and open set. Then

$$
\begin{aligned}
& \text { (compact set of } \left.W^{-1, q}(\Omega)\right) \cap\left(\text { bounded set of } W^{-1, r}(\Omega)\right) \\
& \left.\quad \subset \text { (compact set of } W_{\text {loc }}^{-1,2}(\Omega)\right),
\end{aligned}
$$

where $q$ and $r$ are constants, $1<q \leqq 2<r<\infty$.

With Lemma 6.1, we have

Theorem 6.1. Assume that $\left(\rho^{h}, m^{h}\right)$ are the approximate solutions of the Cauchy problem (1.3) and (1.5) or the initial-boundary value problem (1.3) and (1.6) with $A(x) \in C^{2}$ and $A(x) \geqq c_{0}>0$. Then the measure sequence

$$
\eta\left(v^{h}\right)_{t}+q\left(v^{h}\right)_{x}
$$


lies in a compact subset of $H_{\mathrm{loc}}^{-1}(\Omega)$ for all weak pairs $(\eta, q)$, where $\Omega \subset \Pi_{T}$ is any bounded and open set.

Proof. For simplicity we will drop the index $h$ of the approximate solutions $v^{h}(x, t)$ and will prove only for the Cauchy problem (1.5) within this proof.

Step 1. For any function $\phi \in C_{0}^{1}\left(\Pi_{T}\right)$, the entropy dissipation measures can be written in the form

$$
\iint_{0 \leqq t \leqq T=m \Delta t}\left(\eta(v) \phi_{t}+q(v) \phi_{x}\right) d x d t=L(\phi)+M(\phi)+N(\phi)+\sum(\phi)+E(\phi),
$$

where

$$
\begin{aligned}
M(\phi) & =\int_{-\infty}^{\infty} \phi(x, T) \eta(v(x, T)) d x-\int_{-\infty}^{\infty} \phi(x, 0) \eta\left(v_{0}(x, 0)\right) d x, \\
N(\phi) & =-\int_{\Pi} \int_{T} \nabla \eta(v) a(x) g(v) \phi(x, t) d x d t, \\
L(\phi) & =\sum_{j, n} \int_{\left(j-\frac{1}{2}\right) h}^{\left(j+\frac{1}{2}\right) h}\left(\eta\left(v_{-}^{n}\right)-\eta\left(v_{+}^{n}\right)\right) \phi(x, n \Delta t) d x \equiv L_{1}(\phi)+L_{2}(\phi), \\
L_{1}(\phi) & =\sum_{j, n} \phi_{j}^{n} \int_{\left(j-\frac{1}{2}\right) h}^{\left(j+\frac{1}{2}\right) h}\left(\eta\left(v_{-}^{n}\right)-\eta\left(v_{+}^{n}\right)\right) d x, \\
L_{2}(\phi) & =\sum_{j, n} \int_{\left(j-\frac{1}{2}\right) h}\left(\eta\left(v_{-}^{n}\right)-\eta\left(v_{+}^{n}\right)\right)\left(\phi-\phi_{j}^{n}\right) d x . \\
\sum(\phi) & =\int_{0}^{T} \sum(\sigma[\eta]-[q]) \phi(x(t), t) d t, \\
|E(\phi)| & \leqq C h^{\beta}\|\phi\|_{H^{1}},
\end{aligned}
$$

where $v_{ \pm}^{n}=v(x, n \Delta t \pm 0), \phi_{j}^{n}=\phi(j h, n \Delta t)$, the summation is taken over all discontinuities in $v$ at a fixed time $t, \sigma$ is the propagating speed of the discontinuity, and $E(\phi)$ is the error term including the error in the steady-state solutions and the error near the vacuum in the construction of approximate solutions.

Let $S=(x(t), t)$ denote a discontinuity in $v(x, t),[\eta]$ and $[q]$ denote the jump of $\eta(v(x, t))$ and $q(v(x, t))$ across $S$ from left to right, respectively,

$$
\begin{aligned}
& {[\eta]=\eta(v(x(t)+0, t))-\eta(v(x(t)-0, t)),} \\
& {[q]=q(v(x(t)+0, t))-q(v(x(t)-0, t)) .}
\end{aligned}
$$

Step 2. Since the speeds of propagation of the approximate solutions $v^{h}(x, t)$ are finite, one can assume

$$
\left.\left(\rho_{0}^{h}, m_{0}^{h}\right)\right|_{x \geqq K+\Lambda T}=\left(h^{\beta}, 0\right),
$$


for sufficiently large $K>0$, without loss of generality. This implies

$$
\int_{-\infty}^{\infty} \eta_{*}^{h}\left(\rho^{h}(x, 0), m^{h}(x, 0)\right) d x<\infty
$$

where

$$
\eta_{*}^{h}=\eta_{*}(v)-\eta_{*}(\bar{v})-\nabla \eta_{*}(\bar{v})(v-\bar{v}) \geqq 0,
$$

and corresponding entropy flux

$$
q_{*}^{h}=q_{*}(v)-q_{*}(\bar{v})-\nabla \eta_{*}(\bar{v})(f(v)-f(\bar{v})),
$$

where $\bar{v}=\left(h^{\beta}, 0\right)$.

Noting that $\left.(\rho, m)\right|_{x \geqq \Lambda T+K}=\left(h^{\beta}, 0\right)$, for sufficiently large $K>0$, we substitute $(\eta, q)=\left(\eta_{*}^{h}, q_{*}^{h}\right)$ and $\phi \equiv 1$ in the equality (6.1). Thus

$$
\sum_{n=1}^{m} \int_{-\infty}^{\infty}\left[\eta_{*}^{h n}\right] d x+\int_{0}^{T} \sum\left(\sigma\left[\eta_{*}^{h}\right]-\left[q_{*}^{h}\right]\right) d t \leqq C .
$$

Using Lemmas 3.2-3.7, we have

$$
\begin{aligned}
\sum_{n=1}^{m} \int_{-\infty}^{\infty}\left[\eta_{*}^{h n}\right] d x & \\
= & \sum_{j, n} \int_{\left(j-\frac{1}{2}\right) h}^{\left(j+\frac{1}{2}\right) h}\left(\eta_{*}^{h}\left(v_{-}\right)-\eta_{*}^{h}\left(v_{j}\right)\right) d x-\sum_{j, n} \int_{\left(j-\frac{1}{2}\right) h}^{\left(j+\frac{1}{2}\right) h}\left(\eta_{*}^{h}\left(v_{+}\right)-\eta_{*}^{h}\left(v_{j}\right)\right) d x \\
= & \sum_{j, n} \int_{\left(j-\frac{1}{2}\right) h}^{\left(j+\frac{1}{2}\right) h} \int_{0}^{1}(1-\tau)\left(v_{-}-v_{j}\right)^{\top} \nabla^{2} \eta_{*}\left(v_{j}+\tau\left(v_{-}-v_{j}\right)\right)\left(v_{-}-v_{j}\right) d \tau d x \\
& -\sum_{j, n} \int_{\left(j-\frac{1}{2}\right) h}^{1} \int_{0}^{1}(1-\tau)\left(v_{+}-v_{j}\right)^{\top} \nabla^{2} \eta_{*}\left(v_{j}\right)\left(v_{+}-v_{j}\right) d \tau d x+\mathbf{O}\left(h^{\frac{3}{2}(1-2 \beta)}\right) \\
= & \sum_{j, n} \int_{\left(j+\frac{1}{2}\right) h} \int_{0}^{1}(1-\tau)\left(v_{-}-v_{j}\right)^{\top} \nabla^{2} \eta_{*}\left(v_{j}+\tau\left(v_{-}-v_{j}\right)\right)\left(v_{-}-v_{j}\right) d \tau d x+\mathbf{O}(1) .
\end{aligned}
$$

Using Lemmas 4.1-4.2, we have

$$
\sigma\left[\eta_{*}^{h}\right]-\left[q_{*}^{h}\right] \geqq C h^{\frac{3}{2}-2 \beta},
$$

that is,

$$
\int_{0}^{T} \sum\left(\sigma\left[\eta_{*}^{h}\right]-\left[q_{*}^{h}\right]\right) d t \geqq-C T h^{\frac{1}{2}-2 \beta} \geqq-C T h^{\frac{1}{6}},
$$

for the convex entropy $\eta_{*}$. We have from (6.9)-(6.11) that

$$
\int_{0}^{T} \sum\left(\sigma\left[\eta_{*}\right]-\left[q_{*}\right]\right) d t=\int_{0}^{T} \sum\left(\sigma\left[\eta_{*}^{h}\right]-\left[q_{*}^{h}\right]\right) d t \leqq C
$$

$$
\sum_{j, n} \int_{\left(j-\frac{1}{2}\right) h}^{\left(j+\frac{1}{2}\right) h} \int_{0}^{1}(1-\tau)\left(v_{-}-v_{j}\right)^{\top} \nabla^{2} \eta_{*}\left(v_{j}+\tau\left(v_{-}-v_{j}\right)\right)\left(v_{-}-v_{j}\right) d \tau d x \leqq C
$$


In particular, since $\nabla^{2} \eta_{*}(r, r) \geqq c_{0}(r, r), c_{0}>0$ constant, one has

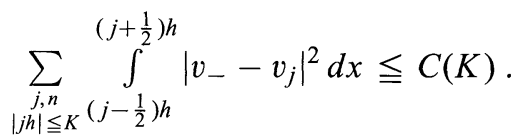

Step 3 For any bounded set $\Omega \subset \Pi_{T}$ and weak entropy pair $(\eta, q)$, we derive from (6.1), (6.4)-(6.5), (6.7), (6.12)-(6.13), and Lemma 2.2 that

$$
\begin{aligned}
&|M(\phi)| \leqq C\|\phi\|_{C_{0}(\Omega)}, \quad|N(\phi)| \leqq C\|\phi\|_{C_{0}(\Omega)}, \\
&\left|\sum(\phi)\right| \leqq C\|\phi\|_{C_{0}(\Omega)} \int_{0}^{T}\left(h^{2(1-\beta)}+\sum\left(\sigma\left[\eta_{*}\right]-\left[q_{*}\right]\right)\right) d x \leqq C\|\phi\|_{C_{0}(\Omega)}, \\
&\left|L_{1}(\phi)\right| \leqq\left|\sum_{j, n} \phi_{j}^{n} \int_{\left(j-\frac{1}{2}\right) h}^{\left(j+\frac{1}{2}\right) h}\left(\eta\left(v_{-}\right)-\eta\left(v_{j}\right)\right) d x\right|+\left|\sum_{j, n} \phi_{j}^{n} \int_{\left(j-\frac{1}{2}\right) h}^{\left(j+\frac{1}{2}\right) h}\left(\eta\left(v_{+}\right)-\eta\left(v_{j}\right)\right) d x\right| \\
& \leqq\|\phi\|_{C_{0}(\Omega)}\left\{\begin{array}{l}
\sum_{j, n} \int_{\left(j-\frac{1}{2}\right) h}^{\left(j+\frac{1}{2}\right) h} \int_{0}^{1}(1-\tau) \mid\left(v_{-}-v_{j}\right)^{\top} \nabla^{2} \eta \\
\times\left(v_{j}+\tau\left(v_{-}-v_{j}\right)\right)\left(v_{-}-v_{j}\right) \mid d \tau d x \\
+\sum_{j, n} \int_{\left(j-\frac{1}{2}\right) h}^{\left(j+\frac{1}{2}\right) h} \int_{0}^{1}(1-\tau) \mid\left(v_{+}-v_{j}\right)^{\top} \nabla^{2} \eta \\
\times\left(v_{j}\right)\left(v_{+}-v_{j}\right) \mid d \tau d x+\mathbf{O}\left(h^{\frac{3}{2}-3 \beta}\right)
\end{array}\right\} \\
& \leqq C\|\phi\|_{C_{0}(\Omega)}\left\{\begin{array}{l}
\sum_{j, n} \int_{\left(j-\frac{1}{2}\right) h}^{\left(j+\frac{1}{2}\right) h} \int_{0}^{1}(1-\tau)\left(v_{-}-v_{j}\right)^{\top} \\
\left.\times \nabla^{2} \eta_{*}\left(v_{j}+\tau\left(v_{-}-v_{j}\right)\right)\left(v_{-}-v_{j}\right) d \tau d x+\mathbf{O}(1)\right\} \\
\leqq
\end{array}\right. \\
& \qquad \|_{C_{0}(\Omega)},
\end{aligned}
$$

where the constant $C$ depends only on the support of $\phi$. Hence

$$
\left|\left(M+N+L_{1}+\sum\right)(\phi)\right| \leqq C\|\phi\|_{C_{0}},
$$

that is

$$
\left\|M+N+L_{1}+\sum\right\|_{C_{0}^{*}} \leqq C
$$

Therefore

$$
M+N+L_{1}+\sum \text { compact in } W^{-1, q_{1}}(\Omega),
$$

where $1<q_{1}<2$. 
Furthermore, for any $\phi \in C_{0}^{\alpha}(\Omega), \frac{1}{2}<\alpha<1$, we have

$$
\begin{aligned}
\left|L_{2}(\phi)\right| & \leqq \sum_{j, n} \int_{\left(j-\frac{1}{2}\right) h}^{\left(j+\frac{1}{2}\right) h}\left|\phi(x, n \Delta t)-\phi_{j}^{n}\right|\left(\left|\eta\left(v_{-}\right)-\eta\left(v_{j}\right)\right|+\left|\eta\left(v_{+}\right)-\eta\left(v_{j}\right)\right|\right) d x \\
& \leqq h^{\alpha}\|\phi\|_{C_{0}^{\alpha}}\left\{\sum_{n}\left(\sum_{j} \int_{\left(j-\frac{1}{2}\right) h}^{\left(j+\frac{1}{2}\right) h}\left|\eta\left(v_{-}\right)-\eta\left(v_{j}\right)\right|^{2} d x\right)^{\frac{1}{2}}+\mathbf{O}(h)\right\} \\
& \leqq C h^{\alpha-\frac{1}{2}}\|\nabla \eta\|_{L^{\infty}}\|\phi\|_{C_{0}^{\alpha}}\left\{\left(\sum_{j, n} \int_{\left(j-\frac{1}{2}\right) h}^{\left(j+\frac{1}{2}\right) h}\left|v_{-}-v_{j}\right|^{2} d x\right)^{\frac{1}{2}}+\mathbf{O}(h)\right\} \\
& \leqq 2 C h^{\alpha-\frac{1}{2}}\|\phi\|_{C_{0}^{\alpha}(\Omega)} .
\end{aligned}
$$

Using the Sobolev theorem: $W_{0}^{1, p}(\Omega) \subset C_{0}^{\alpha}(\Omega), 0<\alpha<1-\frac{2}{p}$, one has

$$
\left|L_{2}(\phi)\right| \leqq C h^{\alpha-\frac{1}{2}}\|\phi\|_{W_{0}^{1, p}(\Omega)}, \quad p>\frac{2}{1-\alpha},
$$

that is

$$
\left\|L_{2}\right\|_{W^{-1, q_{2}(\Omega)}} \leqq C h^{\alpha-\frac{1}{2}} \rightarrow 0, \quad h \rightarrow 0
$$

for $1<q_{2}<\frac{2}{1+\alpha}$.

Moreover,

$$
\|E\|_{H^{-1}} \leqq C h^{\beta} \rightarrow 0, \quad \text { as } h \rightarrow 0 .
$$

It follows from $(6.15)-(6.17)$ that

$$
M+N+L+\sum+E \text { compact in } W_{\mathrm{loc}}^{-1, q_{0}},
$$

where $1<q_{0} \equiv \min \left(q_{1}, q_{2}\right)<\frac{2}{1+\alpha}$.

The fact $0 \leqq \rho \leqq C$ and $\left|\frac{m}{\rho}\right| \leqq C$ implies

$$
M+L+N+\sum+E \text { bounded in } W_{\mathrm{loc}}^{-1, r}(r>1) .
$$

We derive from (6.17)-(6.19) and Lemma 6.1 that

$$
M+L+N+\sum \text { compact in } H_{\mathrm{loc}}^{-1} .
$$

This means that

$$
\eta\left(v^{h}\right)_{t}+q\left(v^{h}\right)_{x} \quad \text { compact in } H_{\mathrm{loc}}^{-1}(\Omega) .
$$

This completes the proof of Theorem 6.1.

\section{Convergence and Consistency}

In Sect. 5 and Sect. 6 , it is proved that the approximate solutions $\left(\rho^{h}, m^{h}\right)$ of the Cauchy problem (1.3) and (1.5), and the initial-boundary problem (1.3) and (1.6) 
satisfy the following conditions:

(1) There is a constant $C(T)>0$ such that

$$
0 \leqq \rho^{h}(x, t) \leqq C, \quad\left|\frac{m^{h}(x, t)}{\rho^{h}(x, t)}\right| \leqq C ;
$$

(2) The measure

$$
\eta\left(v^{h}\right)_{t}+q\left(v^{h}\right)_{x} \text { is compact in } H_{\mathrm{loc}}^{-1}(\Omega),
$$

for all weak entropy pairs $(\eta, q)$, where $\Omega \subset \Pi_{T}$ is any bounded and open set.

The compensated compactness framework (see [C1, C2]) ensures the strong compactness of the approximate solutions $v^{h}(x, t)$ in $L_{\text {loc }}^{1}\left(\Pi_{T}\right)$ for $1<\gamma \leqq 5 / 3$.

Theorem 7.1. Assume that $\left(\rho^{h}, m^{h}\right)$ are the approximate solutions of the Cauchy problem (1.3) and (1.5) with $a(x) \in C^{1}(\mathbf{R})$, or the initial-boundary problem (1.3) and (1.6) with $a(x) \in C^{1}[1, \infty)$, satisfying the conditions (7.1)-(7.2). Then there is a convergent subsequence in the approximations $\left(\rho^{h}(x, t), m^{h}(x, t)\right)$ such that

$$
\left(\rho^{h_{n}}(x, t), m^{h_{n}}(x, t)\right) \rightarrow(\rho(x, t), m(x, t)), \quad \text { a.e. }
$$

The pair of functions $(\rho(x, t), m(x, t))$ is a global entropy solution of the Cauchy problem (1.3) and (1.5) or the initial-boundary problem (1.3) and (1.6) in the sense of Definition 1.1 and satisfies

$$
0 \leqq \rho(x, t) \leqq C(T), \quad\left|\frac{m(x, t)}{\rho(x, t)}\right| \leqq C(T)
$$

in the region $\Pi_{T}$ for any fixed $T \in(0, \infty)$.

Proof. It suffices to prove the limit functions $(\rho, m)$ satisfy $(1.11)-(1.12)$. We prove this only for the approximate solutions $v^{h}$ of the Cauchy problem (1.3) and (1.5). The proof for the approximate solutions of the initial-boundary problem (1.3) and (1.6) is the same.

Notice that for any convex weak entropy pair $(\eta, q)$ and any nonnegative test function $\phi \in C_{0}^{1}\left(\Pi_{T}\right)$,

$$
\begin{aligned}
& \iint_{0 \leqq t \leqq T=m \Delta t}\left(\eta\left(v^{h}\right) \phi_{t}+q\left(v^{h}\right) \phi_{x}+a(x) \nabla \eta\left(v^{h}\right) g\left(v^{h}\right) \phi\right) d x d t+\int_{-\infty}^{\infty} \eta\left(v_{0}^{h}(x)\right) \phi(x, 0) d x \\
= & \sum_{j, n} \phi_{j}^{n} \int_{\left(j-\frac{1}{2}\right) h}^{\left(j+\frac{1}{2}\right) h}\left(\eta\left(v_{-}^{h n}\right)-\eta\left(v_{+}^{h n}\right)\right) d x+\sum_{j, n} \int_{\left(j-\frac{1}{2}\right) h}^{\left(j+\frac{1}{2}\right) h}\left(\eta\left(v_{-}^{h n}\right)-\eta\left(v_{+}^{h n}\right)\right)\left(\phi-\phi_{j}^{n}\right) d x \\
& +\int_{0}^{T} \sum(\sigma[\eta]-[q]) \phi(x(t), t) d t+E(\phi)
\end{aligned}
$$

where $v_{-}^{h n}=v^{h}(x, n \Delta t-0), \phi_{j}^{n}=\phi(j h, n \Delta t)$, the summation is taken over all discontinuities in $v$ at a fixed time $t, \sigma$ is the propagating speed of the discontinuity, and

$$
|E(\phi)| \leqq C h^{\beta}\|\phi\|_{H^{1}} \text {. }
$$


Noting from Lemmas 4.1 and 4.2 that

$$
\sigma[\eta]-[q] \geqq-C h^{\frac{3}{2}-2 \beta}
$$

for the convex entropy $\eta$, one has

$$
\int_{0}^{T} \sum(\sigma[\eta]-[q]) \phi(x(t), t) d t \geqq-C h^{\frac{1}{2}(1-4 \beta)}\|\phi\|_{C} .
$$

Furthermore, for any $\phi \in C_{0}^{1}(\Omega)$, we have,

$$
\begin{aligned}
& \sum_{j, n} \phi_{j}^{n} \int_{\left(j-\frac{1}{2}\right) h}^{\left(j+\frac{1}{2}\right) h}\left(\eta\left(v_{-}^{h}\right)-\eta\left(v_{+}^{n}\right)\right) d x \\
& =\sum_{j, n} \phi_{j}^{n} \int_{\left(j-\frac{1}{2}\right) h}^{\left(j+\frac{1}{2}\right) h} \int_{0}^{1}(1-\tau)\left(v_{-}^{h}-v_{j}^{n}\right)^{\top} \nabla^{2} \eta\left(v_{j}^{n}+\tau\left(v_{-}^{h}-v_{j}^{n}\right)\right)\left(v_{-}^{h}-v_{j}^{n}\right) d \tau d x \\
& \quad-\sum_{j, n} \phi_{j}^{n} \int_{\left(j-\frac{1}{2}\right) h}^{\left(j+\frac{1}{2}\right) h} \int_{0}^{1}(1-\tau)\left|\left(v_{+}^{h}-v_{j}^{n}\right)^{\top} \nabla^{2} \eta\left(v_{j}^{n}\right)\left(v_{+}^{h}-v_{j}^{n}\right)\right| d \tau d x+\mathbf{O}\left(h^{\frac{1}{2}(1-6 \beta)}\right) \\
& \geqq-C\|\phi\|_{C_{0}}\left(\left\|\rho \nabla^{2} \eta(v)\right\|_{L^{\infty}}+h^{\frac{1}{2}(1-6 \beta)}\right),
\end{aligned}
$$

and

$$
\begin{aligned}
& \sum_{j, n} \int_{\left(j-\frac{1}{2}\right) h}^{\left(j+\frac{1}{2}\right) h}\left|\phi(x, n \Delta t)-\phi_{j}^{n}\right|\left(\left|\eta\left(v_{-}^{h}\right)-\eta\left(v_{j}^{n}\right)\right|+\left|\eta\left(v_{+}\right)-\eta\left(v_{j}^{n}\right)\right|\right) d x \\
& \leqq h\|\phi\|_{C_{0}^{1}}\left\{\sum_{n}\left(\sum_{j} \int_{\left(j-\frac{1}{2}\right) h}^{\left(j+\frac{1}{2}\right) h}\left|\eta\left(v_{-}^{h}\right)-\eta\left(v_{j}^{n}\right)\right|^{2} d x\right)^{\frac{1}{2}}+\mathbf{O}(h)\right\} \\
& \leqq \sqrt{h}\|\nabla \eta\|_{L^{\infty}}\|\phi\|_{C_{0}^{1}}\left\{\left(\sum_{j, n} \int_{\left(j-\frac{1}{2}\right) h}^{\left(j+\frac{1}{2}\right) h}\left|v_{-}^{h}-v_{j}^{n}\right|^{2} d x\right)^{\frac{1}{2}}+\mathbf{O}(h)\right\} \\
& \leqq 2 C \sqrt{h}\|\phi\|_{C_{0}^{1}(\Omega)} \rightarrow 0, \quad \text { as } h \rightarrow 0 .
\end{aligned}
$$

Therefore,

$$
\begin{aligned}
& \quad \iint_{0 \leqq t \leqq T=m \Delta t}\left(\eta\left(v^{h}\right) \phi_{t}+q\left(v^{h}\right) \phi_{x}+a(x) \nabla \eta\left(v^{h}\right) g\left(v^{h}\right) \phi\right) d x d t+\int_{-\infty}^{\infty} \eta\left(v_{0}^{h}(x)\right) \phi(x, 0) d x \\
& \quad \geqq-C\left(\|\phi\|_{C_{0}}\left\|\rho \nabla^{2} \eta(v)\right\|_{L^{\infty}}+h^{\frac{1}{2}(1-6 \beta)}\|\phi\|_{C_{0}^{1}}+h^{\beta}\|\phi\|_{H^{1}}\right) \\
& \quad \rightarrow-C\|\phi\|_{C_{0}}, \quad h \rightarrow 0 .
\end{aligned}
$$

Taking the limit $h \rightarrow 0$ on both of sides of (7.7) and using the Dominated Control Theorem, we verify that the limit function $v=(\rho, m)$ satisfies

$$
\eta(v)_{t}+q(v)_{x}-a(x) \nabla \eta(v) g(v) \leqq C\left\|\rho \nabla^{2} \eta(v)\right\|_{L^{\infty}},
$$


in the sense of distributions. Choosing $(\eta(v), q(v))=( \pm v, \pm f(v))$, we immediately conclude that $v(x, t)$ is a weak solution. Using the standard procedure (cf. [La1, $\mathrm{D} 2, \mathrm{Sm}])$, we conclude that the limit function $v(x, t)$ satisfies the entropy condition (1.12) along any shock wave. This completes the proof of Theorem 7.1.

\section{Transonic Nozzle Flow: Proof of Theorem A}

For the transonic nozzle flow, $A(x)$ represents the cross-sectional area of the nozzle at $x$. The first equation in (1.2) comes from the conservation of mass in the form

$$
(\rho A)_{t}+(m A)_{x}=0,
$$

and the second equation in (1.2) is the conservation of momentum that can be rewritten as

$$
(m A)_{t}+\left(\frac{m^{2}}{\rho} A\right)_{x}+A p(\rho)_{x}=0 .
$$

For the system (8.1) and (8.2), or more generally (1.3), we have constructed the approximate solutions for the Cauchy problem (1.3) and (1.5) in Sect. 4 and have proved that the approximate solutions satisfy the conditions (7.1) and (7.2) of Theorem 7.1 in Sects. 5 and 6, provided that $A(x)$ is a $C^{2}$ function bounded away from zero for all $x \in \mathbf{R}$ and the initial velocity and nonnegative density data $\left(\frac{m_{0}}{\rho_{0}}, \rho_{0}\right)$ are bounded in $L^{\infty}$. Using Theorem 7.1, we conclude that there is a subsequence in the approximate solutions strongly converging to the $L^{\infty}$ function $(\rho(x, t), m(x, t))$ almost everywhere and that $(\rho(x, t), m(x, t))$ satisfies (7.4). For any function $\phi \in$ $C_{0}^{1}\left(\mathbf{R}_{+}^{2}\right)$, we define $\psi(x, t)=A(x) \phi(x, t)$, which is still in $C_{0}^{1}\left(\mathbf{R}_{+}^{2}\right)$. In particular, choosing $\eta=\rho$ and $m$ in (7.5) from Theorem 7.1, one has

$$
\int_{0}^{\infty} \int_{-\infty}^{\infty}\left(\rho A(x) \phi_{t}+m A(x) \phi_{x}\right) d x d t=\int_{0}^{\infty} \int_{-\infty}^{\infty}\left(\rho \psi_{t}+m \psi_{x}-\frac{A^{\prime}(x)}{A(x)} m \psi\right) d x d t=0,
$$

and

$$
\begin{aligned}
& \int_{0}^{\infty} \int_{-\infty}^{\infty}\left(m A(x) \phi_{t}+\frac{m^{2}}{\rho} A(x) \phi_{x}+p(\rho)(A(x) \phi)_{x}\right) d x d t \\
& =\int_{0}^{\infty} \int_{-\infty}^{\infty}\left(m \psi_{t}+\left(\frac{m^{2}}{\rho}+p(\rho)\right) \psi_{x}-\frac{A^{\prime}(x)}{A(x)} \frac{m^{2}}{\rho} \psi\right) d x d t=0 .
\end{aligned}
$$

Then Theorem B stated in Sect. 1 follows.

\section{Spherically Symmetric Flow: Proof of Theorem B}

Consider the spherically symmetric flow of the compressible Euler equations (1.1) in $\mathbf{R}^{N}$ :

$$
(\rho(\vec{x}, t), \vec{m}(\vec{x}, t))=\left(\rho(x, t), m(x, t) \frac{\vec{x}}{x}\right),
$$

where $x=|\vec{x}|$ and $m(x, t)$ is a scalar function. The case $N=2$ corresponds to the cylindrically symmetric flows and the case $N \geqq 3$ corresponds to the $N$-dimensional 
spherically symmetric flow. Then we obtain from (1.1) and (9.1) that $(\rho(x, t), m(x, t))$ satisfies (1.2) and (1.4) with $A(x)=\frac{2 \pi^{\frac{N}{2}}}{\Gamma\left(\frac{N}{2}\right)} x^{N-1}$.

Consider the initial-boundary problem for the system of compressible Euler equations (1.1) with initial-boundary values (1.14). We have constructed the approximate solutions for the initial-boundary problem (1.3) and (1.6) in Sect. 4 and have proved that the approximate solutions satisfy the conditions (7.1) and (7.2) of Theorem 7.1 in Sects. 5 and 6, provided that the initial velocity and nonnegative density data $\left(\frac{m_{0}}{\rho_{0}}, \rho_{0}\right)$ are bounded in $L^{\infty}$. Using Theorem 7.1, we conclude that there is a subsequence in the approximate solutions strongly converging to the $L^{\infty}$ function $(\rho(x, t), m(x, t))$ almost everywhere and that $(\rho(x, t), m(x, t))$ satisfies (7.6)-(7.7). Therefore, it suffices to prove that the functions $(\rho(\vec{x}, t), \vec{m}(\vec{x}, t))$, determined by the function $(\rho(x, t), m(x, t))$ through (9.1), satisfy the condition (1)(3) of Definition 1.2.

For any test function $\phi \in C_{0}^{1}(\{|\vec{x}|>1\} \times[0, \infty))$, set $\vec{\phi}(\vec{x}, t)=\phi(x, t) \frac{\vec{x}}{x} \in C_{0}^{1}$ $(\{|\vec{x}|>1\} \times[0, \infty))$. We have

$$
\begin{aligned}
& \int_{0}^{\infty} \int_{|\vec{x}| \geqq 1}\left(\vec{m} \vec{\phi}_{t}+\frac{\vec{m} \otimes \vec{m}}{\rho} \nabla \vec{\phi}+p(\rho) \operatorname{div} \vec{\phi}\right) d \vec{x} d t+\int_{|\vec{x}| \geqq 1} \vec{m}_{0}(\vec{x}) \vec{\phi}(\vec{x}, 0) d \vec{x} \\
& =\int_{|\vec{x}|=1} \int_{0}^{\infty} \int_{1}^{\infty}\left(m \phi_{t}+\frac{m^{2}}{\rho} \phi_{x}+p(\rho)\left(\phi_{x}+\frac{N-1}{x} \phi\right)\right) x^{N-1} d x d S d t \\
& \quad+\int_{|\vec{x}|=1} \int_{1}^{\infty} m_{0}(x) \phi(x, 0) d x d S \\
& =\int_{0}^{\infty} \int_{1}^{\infty}\left(m A(x) \phi_{t}+\frac{m^{2}}{\rho} A(x) \phi_{x}+p(\rho)(A(x) \phi)_{x}\right) d x d t \\
& \quad+\int_{1}^{\infty} \rho_{0}(x) A(x) \phi(x, 0) d x=0 .
\end{aligned}
$$

Set $\psi(x, t)=\phi(x, t) A(x)$ that is still $C_{0}^{1}((1, \infty) \times[0, \infty))$ function. Thus

$$
\begin{aligned}
& \int_{0}^{\infty} \int_{|\vec{x}| \geqq 1}\left(\vec{m} \vec{\phi}_{t}+\frac{\vec{m} \otimes \vec{m}}{\rho} \nabla \vec{\phi}+p(\rho) \operatorname{div} \vec{\phi}\right) d \vec{x} d t+\int_{|\vec{x}| \geqq 1} \vec{m}_{0}(\vec{x}) \vec{\phi}(\vec{x}, 0) d \vec{x} \\
& \quad=\int_{0}^{\infty} \int_{1}^{\infty}\left(m \psi_{t}+\frac{m^{2}}{\rho} \psi_{x}+p(\rho) \psi_{x}-\frac{A^{\prime}(x)}{A(x)} \frac{m^{2}}{\rho} \psi\right) d x d t+\int_{1}^{\infty} m_{0}(x) \psi(x, 0) d x=0 .
\end{aligned}
$$

Similarly, for any test function $\phi \in C_{0}^{1}(\{|\vec{x}|>1\} \times[0, \infty))$ satisfying $\phi(\vec{x}, t)=$ $\phi(x, t)$, we set $\psi(x, t)=\phi(x, t) A(x)$ that is still $C_{0}^{1}((1, \infty) \times[0, \infty))$ function in the identity:

$$
\int_{0}^{\infty} \int_{1}^{\infty}\left(\rho \psi_{t}+m \psi_{x}-\frac{A^{\prime}(x)}{A(x)} m \psi\right) d x d t+\int_{1}^{\infty} \rho_{0}(x) \psi(x, 0) d x=0
$$

and conclude the condition (1) of Definition 1.2. 
For (1.16), for any nonnegative test function $\phi \in C_{0}^{1}(\{|\vec{x}|>1\} \times(0, \infty))$ satisfying $\phi(\vec{x}, t)=\phi(x, t)$, we have

$$
\begin{gathered}
\int_{0}^{\infty} \int_{|\vec{x}| \geqq 1}\left(\rho\left(\frac{1}{2} \frac{|\vec{m}|^{2}}{\rho^{2}}+e\right) \phi_{t}+\vec{m}\left(\frac{1}{2} \frac{|\vec{m}|^{2}}{\rho^{2}}+e+\frac{p}{\rho}\right) \cdot \nabla \phi\right) d \vec{x} d t \\
=\int_{|\vec{x}|=1} \int_{0}^{\infty} \int_{1}^{\infty}\left(\left(\frac{1}{2} \frac{m^{2}}{\rho}+\rho e\right) \phi_{t}+m\left(\frac{1}{2} \frac{m^{2}}{\rho^{2}}+e+\frac{p}{\rho}\right) \phi_{x}\right) x^{N-1} d x d t d S \\
=\int_{0}^{\infty} \int_{1}^{\infty}\left(\left(\frac{1}{2} \frac{m^{2}}{\rho}+\rho e\right) \phi_{t}+m\left(\frac{1}{2} \frac{m^{2}}{\rho^{2}}+e+\frac{p}{\rho}\right) \phi_{x}\right) A(x) d x d t \\
=\int_{0}^{\infty} \int_{1}^{\infty}\left(\left(\frac{1}{2} \frac{m^{2}}{\rho}+\rho e\right) \psi_{t}+m\left(\frac{1}{2} \frac{m^{2}}{\rho^{2}}+e+\frac{p}{\rho}\right) \psi_{x}\right. \\
\left.\quad-\frac{A^{\prime}(x)}{A(x)} m\left(\frac{1}{2} \frac{m^{2}}{\rho^{2}}+\frac{\rho^{\gamma-1}}{\gamma-1}\right) \psi\right) d x d t \\
\geqq-C\|\phi\|_{C_{0}},
\end{gathered}
$$

using (6.5) with $\eta(v)=\frac{1}{2} \frac{m^{2}}{\rho}+\frac{\rho^{\gamma}}{\gamma(\gamma-1)}$. Using the standard procedure (cf. [La1, D2, $\mathrm{Sm}])$, we conclude that the weak solution $(\rho(\vec{x}, t), \vec{m}(\vec{x}, t))$ satisfies the entropy condition (1.16).

The result (1.15) follows directly from (9.1) and standard techniques as in [MT].

Using completely the same arguments without any difficulties, one can similarly obtain a global solution for the system with a gravitational source

$$
\left\{\begin{array}{l}
\rho_{t}+m_{x}=-\frac{N-1}{x} m, \\
m_{t}+\left(\frac{m^{2}}{\rho}+p(\rho)\right)_{x}=-\frac{N-1}{x} \frac{m^{2}}{\rho}-\frac{\rho M}{x^{2}},
\end{array}\right.
$$

which describes an atmosphere surrounding a solid star with radius 1 and mass $M$. The local solutions have been constructed in [MT].

Acknowledgements. This work was completed when the first author visited the Institute for Advanced Study He would like to thank Luis Caffarelli and Thomas Spencer for their hospitality and stimulating conversations Gui-Qiang Chen was supported in part by the Office of Naval Research grant N00014-91-J-1384, the National Science Foundation grants DMS-9623203 and DMS9201581, by an Alfred P. Sloan Foundation Fellowship, and by an Alfred P. Sloan Foundation Grant No. 3126 through the Institute for Advanced Study. James Glimm was supported in part by the Applied Mathematics Subprogram of the U.S. Department of Energy DE-FG02-90ER25084, by the Army Research Office grant DAAL03-92-G-0185 through the Mathematical Sciences Institute of Cornell University under subcontract to the University at Stony Brook, ARO contract number DAAH049510414, and by the National Science Foundation grant DMS-9500568

\section{References}

[C1] Chen, G-Q.: The compensated compactness method and the system of isentropic gas dynamics. MSRI Preprint 00527-91, Berkeley (1990)

[C2] Chen, G-Q : Convergence of the Lax-Friedrichs scheme for isentropic gas dynamics (III) Acta Mathematica Scientia 8, 243-276 (1988) (in Chinese); 6, 75-120 (1986) (in English) 
[C3] Chen, G.-Q : Remarks on spherically symmetric solutions to the compressible Euler equations. Proc Royal Soc Edinburgh, 1996 (to appear)

[CG] Chen, G-Q and Glimm, J : Global solutions to the cylindrically symmetric rotating motion of isentropic gas Z Angew Math. Phys 1996 (to appear)

[CW] Chen, G -Q and Wang, D : Convergence of shock capturing schemes for the compressible Euler-Poisson equations Commun Math Phys 1996 (to appear)

[CF] Courant, R and Friedrichs, K.O : Supersonic flow and shock waves New York, SpringerVerlag, 1948

[D1] Dafermos, C : Polygonal approximations of solutions of the initial-value problem for a conservation law J Math Anal Appl 38, 33-41 (1972)

[D2] Dafermos, C : Hyperbolic Systems of Conservation Laws In: Systems of Nonlinear Partial Differential Equations, NATO Adv Sci Inst Ser C: Math Phys Sci 111, 25-70 (1983)

[DC1] Ding, X., Chen, G.-Q and Luo, P.: Convergence of the Lax-Friedrichs scheme for isentropic gas dynamics (I)-(II) Acta Mathematica Scientia 7, 467-480 (1987), 8, 61-94 (1988) (in Chinese); 5, 415-432, 433-472 (in English)

[DC2] Ding, X, Chen, G-Q. and Luo, P : Convergence of the fractional step Lax-Friedrichs scheme and Godunov scheme for the isentropic system of gas dynamics Commun Math Phys 121, 63-84 (1989)

[Di] DiPerna, R : Convergence of the viscosity method for isentropic gas dynamics Commun Math Phys 91, 1-30 (1983)

[EM] Embid, P, Goodman, J and Majda, A.: Multiple steady state for 1-D transonic flow SIAM J Sci. Stat Comp. 5, 21-41 (1984)

[Fo] Fok, S K : Extensions of Glimm's method to the problem of gas flow in a duct of variable cross-section Ph.D. Thesis, Department of Mathematics, University of California at Berkeley, 1980

[GL] Glaz, $\mathrm{H}$ and Liu, T.-P.: The asymptotic analysis of wave interactions and numerical calculation of transonic nozzle flow Adv Appl Math 5, 111-146 (1984)

[Gl] Glimm, J : Solutions in the large for nonlinear hyperbolic systems of equations Commun Math. Phys 18, 697-715 (1965)

[GM] Glimm, J, Marshall, G and Plohr, B : A generalized Riemann problem for quasi-onedimensional gas flow. Adv Appl Math 5, 1-30 (1984)

[Ho] Hoff, D : Spherically symmetric solutions of the Navier-Stokes equations for compressible, isothermal flow with large, discontinuous initial data Indiana U. Math. J 41, 1225-1302 (1992)

[La1] Lax, P D : Hyperbolic Systems of Conservation Laws and the Mathematical Theory of Shock Waves CBMS 11, SIAM, 1973

[La2] Lax, P D : Weak solutions of nonlinear hyperbolic equations and their numerical computation. Commun Pure Appl Math 7, 159-193 (1954)

[LP] Lions, P L, Perthame, B and Tadmor, E: Kinetic formulation of the isentropic gas dynamics and $p$-systems Commun Math Phys 163, 169-192 (1994)

[L1] Liu, T -P : Quasilinear hyperbolic systems Commun Math Phys 68, 141-172 (1979)

[L2] Liu, T.-P : Nonlinear stability and instability of trasonic gas flow through a nozzle Commun. Math. Phys 83, 243-260 (1983)

[L3] Liu, T.-P.: Nonlinear resonance for quasilinear hyperbolic equation J. Math Phys 28, 2593-2602 (1987)

[MU] Makino, T., Mizohata, K and Ukai, S : Global weak solutions of the compressible Euler equations with spherical symmetry I, II Japan J Industrial Appl Math 9, 431-449 (1992)

[MT] Makino, T and Takeno, S : Initial-Boundary Value Problem for the spherically symmetric motion of isentropic gas Japan J. Industrial Appl Math 11, 171-183 (1994)

[M] Morawetz, C.S.: On a weak solution for a transonic flow problem Commun Pure Appl. Math. 38, 797-818 (1985)

[Mu Murat, F : L'injection du cone positif de $H^{-1}$ dans $W^{-1, q}$ est compacte pour tout $q<2$ J Math Pures Appl 60, 309-322 (1981)

[OM] Okada, $\mathrm{M}$ and Makino, T : Free boundary problem for the equation of spherically symmetric motion of viscous gas Japan J Indust Appl Math (to appear) (1994)

[Se] Serre, D : La compacité par compensation pour les systèmes hyperboliques non linéaires de deux équations à une dimension d'espace J Math Pures Appl. 65, 423-468 (1986)

[Sl] Slemrod, M.: Resolution of the spherical piston problem for compressible isentropic gas dynamics via a self-similar viscous limit Proc Royal Soc. Edinburgh 1996 (to appear) 
[Sm] Smoller, J : Shock Waves and Reaction-Diffusion Equations New York: Springer-Verlag, 1983

[Ta] Tartar, L: Compensated compactness and applications to partial differential equations Research Notes in Mathematics, Nonlinear Analysis and Mechanics, ed R J Knops, Vol 4, New York: Pitman Press, 1979

[Wh] Whitham, G B.: Linear and Nonlinear Waves. New York: John Wiley and Sons, 1974

Communicated by A Jaffe 
